\title{
New Horizon: On the Origin of the Stellar Disk and Spheroid of Field Galaxies at $z=0.7$
}

\author{
Min-Jung Park ${ }^{1}$ (1), Sukyoung K. Yi ${ }^{1}$ (D), Yohan Dubois ${ }^{2}$, Christophe Pichon ${ }^{2,3}$, Taysun Kimm ${ }^{1}$ (1), Julien Devriendt ${ }^{4}$, \\ Hoseung Choi $^{5}$ (D), Marta Volonteri ${ }^{2}$ (D), Sugata Kaviraj ${ }^{6}$, and Sebastien Peirani ${ }^{7}$ \\ ${ }^{1}$ Department of Astronomy and Yonsei University Observatory, Yonsei University, Seoul 03722, Republic of Korea \\ ${ }^{2}$ Institut d'Astrophysique de Paris, Sorbonne Universités, UMPC Univ. Paris 06 et CNRS, UMP 7095, 98 bis bd Arago, F-75014 Paris, France \\ ${ }^{3}$ Korea Institute of Advanced Studies (KIAS), 85 Hoegiro, Dongdaemun-gu, Seoul, 02455, Republic of Korea \\ ${ }^{4}$ Dept. of Physics, University of Oxford, Keble Road, Oxford OX1 3RH, UK \\ ${ }^{5}$ Institute of Theoretical Astrophysics, University of Oslo, Postboks 1029, Blindern, NO-0315 Oslo, Norway \\ ${ }^{6}$ Centre for Astrophysics Research, School of Physics, Astronomy and Mathematics, University of Hertfordshire, College Lane, Hatfield, AL10 9AB, UK \\ ${ }^{7}$ Observatoire de la Côte d'Azur, CNRS, Laboratoire Lagrange, Bd de l'Observatoire, Université Côte d'Azur, CS 34229, F-06304 Nice Cedex 4, France \\ Received 2019 May 1; revised 2019 July 21; accepted 2019 August 12; published 2019 September 18
}

\begin{abstract}
The origin of the disk and spheroid of galaxies has been a key open question in understanding their morphology. Using the high-resolution cosmological simulation New Horizon, we explore kinematically decomposed disk and spheroidal components of 144 field galaxies with masses greater than $10^{9} M_{\odot}$ at $z=0.7$. The origins of stellar particles are classified according to their birthplace (in situ or ex situ) and their orbits at birth. Before disk settling, stars form mainly through chaotic mergers between protogalaxies and become part of the spheroidal component. When disk settling starts, we find that more massive galaxies begin to form disk stars from earlier epochs; massive galaxies commence to develop their disks at $z \sim 1-2$, while low-mass galaxies do after $z \sim 1$. The formation of disks is affected by accretion as well, as mergers can trigger gas turbulence or induce misaligned gas infall that hinders galaxies from forming corotating disk stars. The importance of accreted stars is greater in more massive galaxies, especially in developing massive spheroids. A significant fraction of the spheroids come from the disk stars that are perturbed, and this becomes more important at lower redshifts. Some $(\sim 12.5 \%)$ of our massive galaxies develop counter-rotating disks from the gas infall misaligned with the existing disk plane, which can last for more than a gigayear until they become the dominant component and flip the angular momentum of the galaxy in the opposite direction. The final disk-to-total ratio of a galaxy needs to be understood in relation to its stellar mass and accretion history. We quantify the significance of the stars with different origins and provide them as guiding values.
\end{abstract}

Key words: galaxies: evolution - galaxies: formation - galaxies: kinematics and dynamics - galaxies: structure

\section{Introduction}

In the local universe, galaxies have a wide variety of morphology ranging from disk-dominated spiral galaxies to bulge-dominated elliptical galaxies (Hubble 1926). From many observational results, it is also well established that the morphology of galaxies is highly correlated with other properties, such as luminosity (mass), color, and star formation rate (e.g., Conselice 2006; Driver et al. 2006; Benson et al. 2007; Ilbert et al. 2010). The two most distinctive components of a galaxy behind this morphological diversity are supposedly their disk and spheroidal components, and their distinct stellar populations and kinematics suggest that these components were formed through different mechanisms. Therefore, identifying the origin of these structures is an important step toward understanding the formation of galaxies with different shapes.

The conventional view of disk formation is that stellar disks are formed when shock-heated gas slowly cools down and collapses into dark matter (DM) haloes while conserving its angular momentum (Fall 1980; Mo et al. 1998; Cole et al. 2000). Recent hydrodynamic simulations have further elaborated on this classical understanding by showing that gas can quickly collapse and form filamentary structures before it reaches galactic haloes, the so-called "cold-mode" accretion (Kereš et al. 2005; Ocvirk et al. 2008; Dekel et al. 2009). This cold stream through the cosmic filaments is thought to be a dominant process of gas accretion for low-mass or highredshift galaxies. In addition, because gas accumulates angular momentum as it travels along the filament, gas infalling later would carry higher angular momentum into a galaxy (Kimm et al. 2011; Pichon et al. 2011; Stewart et al. 2011). Continuous supply of gas seems to play an important role in the formation of disk galaxies; for example, gas-rich major mergers are proposed as one possible channel for the formation of massive disk galaxies (e.g., Barnes 2002; Governato et al. 2009).

In a hierarchical universe, galaxies grow into massive ellipticals via mergers. As a result of mergers, galaxies develop dispersion-dominated spheroidal components as the aligned orbits of disk stars are disturbed (Toomre 1972; Negroponte \& White 1983). Therefore, disk-dominated galaxies are generally expected to have experienced fewer violent events that could destroy their disks, and the formation of massive elliptical galaxies is believed to be the result of numerous hierarchical mergers. Accordingly, merger history has been considered as the most important factor in determining the morphology of galaxies, as shown in the results of several numerical studies (e.g., Scannapieco et al. 2009; Martig et al. 2012; Aumer et al. 2014; Martin et al. 2018).

"Accretion" of the stars of disrupted satellite galaxies during the process of mergers is also an important channel in the development of spheroids. These accreted stars are believed to contribute to the growth of the outskirts of galaxies (Oser et al. 2010), and especially to the formation of the halo components (Searle \& Zinn 1978; Zolotov et al. 2009; Tissera et al. 2012, 2013; Cooper et al. 2015). From a hierarchical point of view, more massive galaxies are expected to have more 


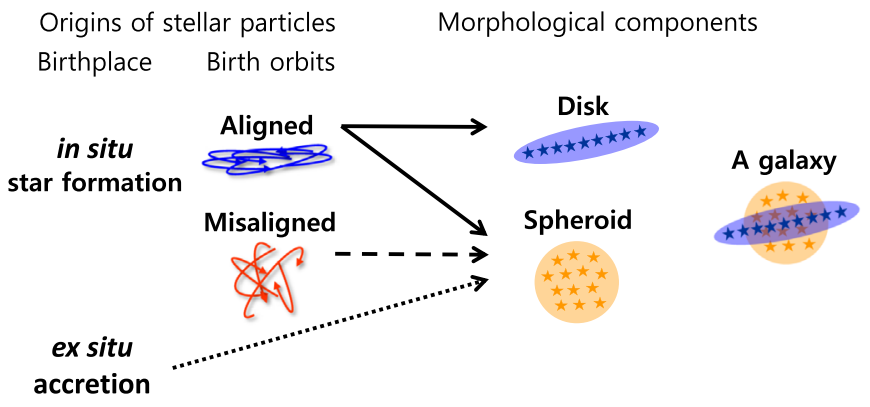

Figure 1. A schematic diagram summarizing the possible channels to the disk and spheroidal components of a galaxy, based on the origins of stellar particles.

accretion (Rodriguez-Gomez et al. 2016; Lee \& Yi 2017), and some studies using cosmological large-volume simulations have argued that accretion plays a major role in driving the morphology of the massive galaxies to be spheroidal (Dubois et al. 2016; Clauwens et al. 2018; Martin et al. 2018). For example, Dubois et al. (2016) have shown that the morphology of galaxies at fixed mass strongly depends on cosmic accretion.

Spheroids can grow through several other processes besides mergers. Many numerical studies have shown that turbulent gas-rich disks at high redshifts tend to fragment into massive clumps as a result of gravitational instabilities; these clumps formed in situ in the protodisks can migrate to the central regions and coalesce to form a central bulge (Noguchi 1999; Elmegreen et al. 2008; Dekel et al. 2009). Another mechanism that contributes to the growth of spheroids is related to the formation of bars; a bar can induce gas inflow into the central regions, enhancing central star formation to form disk-like bulges (Athanassoula 2005).

Misaligned gas infall is also thought to contribute to disk shrinkage and redistribution of mass from disks to spheroids (Scannapieco et al. 2009; Zolotov et al. 2015). Occasionally, a counter-rotating component develops in a galaxy during the misaligned infall, which can cause reorientation of disks. Aumer \& White (2013) found in their simulation that disk fraction decreases as galaxies have experienced more frequent reorientation. Several studies have claimed that the spin alignment of gas accretion is more crucial in determining the final morphology of galaxies than the frequency of mergers (Pichon et al. 2011; Sales et al. 2012). All of the above-mentioned internal and external processes affect the formation of spheroids, but it is still unclear which process is the most significant.

Hence, the origin of disk and spheroid may be speculated as follows. Spheroids form from material with low angular momentum in the early stage of galaxy formation where chaotic mergers between protogalaxies are frequent. Galactic disks develop later with in situ star formation from coherent accretion of gas. As galaxies evolve, new spheroidal components can further grow from disk instability, misaligned gas infall, and hierarchical merging. Therefore, as the schematic diagram of Figure 1 shows, disks are mainly composed of the stars formed in situ with corotating coplanar initial orbits ("aligned" orbits), while spheroids consist of the stars formed in situ with noncoplanar initial orbits ("misaligned" orbits, dashed arrow), as well as the stars with disk-origins (solid arrow), and the stars formed ex situ and later accreted (dotted arrow).

In this study, we use the New Horizon simulation (Y. Dubois et al. 2019, in preparation), a high-resolution cosmological zoom-in simulation that includes a statistically significant number of galaxies, to understand the origin of disk and spheroidal components. This study aims to quantify the relative importance of the channels to the disk and spheroidal components (see Figure 1), as probed by our sample. Specifically, this study attempts to answer the following questions: (1) when do galaxies start to predominantly form disk stars? (2) what is the contribution of each channel to the formation of spheroids? and (3) how does galactic morphology evolve when a galaxy develops a counter-rotating component from gas infall misaligned with the existing disk plane?

This paper is organized as follows. In Section 2, we describe the New Horizon simulation, galaxy identification, and sample selection. Section 3 presents the kinematic decomposition techniques used to identify the disk and spheroidal components and to measure the mass ratio of the components as a morphological indicator. In Section 4, we explore the formation of disk and spheroidal components of the New Horizon galaxies by tracing the origin of stellar particles. In Section 5 , we examine the evolution of kinematic morphology until $z=0.7$ and provide the estimates of the contributions from different channels to the disk and spheroidal components. Finally, we summarize our results in Section 6.

\section{Methodology}

\subsection{The New Horizon Simulation}

We use the New Horizon simulation (Y. Dubois et al. 2019, in preparation), a high-resolution cosmological simulation performed with an Eulerian hydrodynamics code with adaptive mesh refinement (AMR), RAMSES (Teyssier 2002). The parent simulation, Horizon-AGN (Dubois et al. 2014a) is a largevolume simulation (with box size of $100 \mathrm{Mpc} / h$ ) that successfully reproduces galaxies in the various cosmic environments, including 14 galaxy clusters with $M_{\text {vir }}>10^{14} M_{\odot}$. While the maximum spatial resolution of $\sim 1 \mathrm{kpc}$ (with a DM mass resolution of $8 \times 10^{7} M_{\odot}$ and a stellar mass resolution of $\left.2 \times 10^{6} M_{\odot}\right)$ allows us to understand the general evolutionary trends of a large number of galaxies $(\sim 85,000$ galaxies above $10^{9} M_{\odot}$ at $z=0$ ), it is still not sufficient to study the detailed structures of galaxies.

The New Horizon simulation zooms in on a sphere with a radius of $10 \mathrm{Mpc}$ (comoving) in a "field" environment ${ }^{8}$ which is extracted from the Horizon-AGN simulation with a DM mass resolution of $10^{6} M_{\odot}$ and a minimum stellar mass resolution of $10^{4} M_{\odot}$. The simulation has reached $z=0.7$ with maximum spatial resolution of $40 \mathrm{pc}$ (physical scale, at $z=0.7) .{ }^{9}$ Note that this simulation has the highest spatial resolution yet in a cosmological volume. Figure 2 shows the maps of the stellar, DM, and gas distributions and the AMR grid of a sample galaxy in the New Horizon simulation. The simulation adopts cosmological parameters consistent with the WMAP-7 data (Komatsu et al. 2011): Hubble constant $H_{0}=70.4 \mathrm{~km} \mathrm{~s}^{-1} \mathrm{Mpc}^{-1}$, total mass density $\Omega_{m}=0.272$, total baryon density $\Omega_{b}=0.0455$, dark energy density

\footnotetext{
8 The mass of the most massive halo in the New Horizon volume at $z=0.7$ is $\sim 3.9 \times 10^{12} M_{\odot}$. In order to quantify the environment, we counted the 10 nearest neighbors (with masses greater than $10^{9} M_{\odot}$ ) for each galaxy in our sample and divided by the circular enclosed area. Based on this quantitative term, galaxy population can be separated as follows: field galaxies $\left(\Sigma<10 \mathrm{Mpc}^{-2}\right)$, group galaxies $\left(\Sigma \sim 100 \mathrm{Mpc}^{-2}\right)$, and cluster galaxies $\left(\Sigma \sim 1000 \mathrm{Mpc}^{-2}\right.$ ) (e.g., Smith et al. 2005). Given that the median value of the projected surface density for our sample is $\Sigma \sim 5.6 \mathrm{Mpc}^{-2}$ (at $z=0.7$ using 100 random projections), most of our sample seems to be biased toward field galaxies.

9 We are trying to find more computing time to reach $z=0$
} 

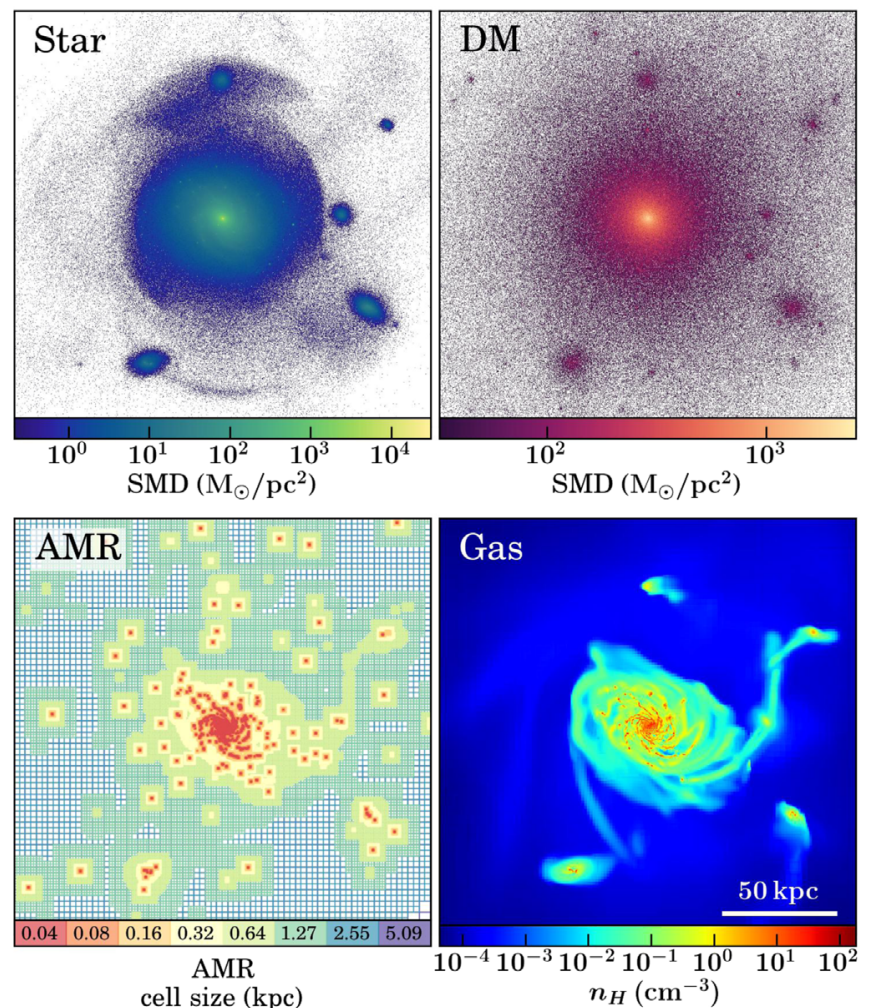

Figure 2. A projected map of the stellar particles, dark matter particles, AMR grid, and gas density of a galaxy at $z=0.7$ in the New Horizon simulation. Note that the minimum mass resolutions for the dark matter and stellar particles are $M_{\mathrm{DM} \text {,res }} \sim 10^{6} M_{\odot}$ and $M_{* \text {,res }} \sim 10^{4} M_{\odot}$, respectively. The maximum spatial resolution is $40 \mathrm{pc}$ (physical scale).

$\Omega_{\Lambda}=0.728$, amplitude of power spectrum $\sigma_{8}=0.809$, and power spectral index $n_{s}=0.967$.

Radiative cooling is modeled following Sutherland \& Dopita (1993) and Rosen \& Bregman (1995); this allows gas to cool to $1 \mathrm{~K}$ through primordial and metal cooling. Gas can also be heated via uniform ultraviolet radiation after the reionization epoch at $z=10$ based on Haardt \& Madau (1996). Stars can form out of gas cells with a hydrogen number density greater than $n_{\mathrm{H}}=10 \mathrm{~cm}^{-3}$ and a temperature lower than $2 \times 10^{4} \mathrm{~K}$, following the Schmidt law (Schmidt 1959). Instead of adopting a fixed star formation efficiency per freefall time $\left(\epsilon_{\mathrm{ff}}\right)$, we determine the local $\epsilon_{\mathrm{ff}}$ based on the local thermo-turbulent condition (Kimm et al. 2017). When each stellar particle becomes older than $5 \mathrm{Myr}$, we assume a supernova explosion returning $31 \%$ of the stellar mass to the surroundings. Supernova feedback is modeled using a mechanical feedback scheme (Kimm \& Cen 2014). We adopt the Chabrier initial mass function (Chabrier 2005) with lower and upper mass cutoffs of $0.1 M_{\odot}$ and $150 M_{\odot}$, respectively.

Black holes, implemented as sink particles, form in cells where both the gas and stellar densities are above the threshold of star formation, with a seed mass of $10^{4} M_{\odot}$. The black holes grow on the basis of Bondi-Hoyle-Lyttleton accretion (Hoyle \& Lyttleton 1939; Bondi \& Hoyle 1944), and the maximum accretion rate is limited to the Eddington rate. Feedback from active galactic nuclei is modeled in two different ways depending on the ratio of the gas accretion rate to the Eddington limit by following Dubois et al. (2012). If the gas accretion rate is lower than $1 \%$ of the Eddington rate, the black hole releases mass, momentum, and energy in the form of jets (radio mode).
The jet efficiency depends on the spin of the black hole that is evolved with the model of Dubois et al. (2014b), with a spin-up rate and an efficiency for the jet mode following magnetically arrested disk simulations (Mckinney et al. 2012). Conversely, if the gas accretion rate is higher than $1 \%$ of the Eddington rate, the black hole deposits thermal energy isotropically (quasar mode). A more detailed description of the simulation can be found in Y. Dubois et al. (2019, in preparation).

\subsection{Galaxy and Halo Identification and Sample Selection}

Simulated galaxies are identified using the ADAPTAHOP algorithm (Aubert et al. 2004) with the most massive sub-node mode (Tweed et al. 2009) applied for stellar particles. At least 50 stellar particles are required to be identified as a galaxy, and the position of the stellar particle with the highest spatial density is considered to be the center of the galaxy. DM haloes are also identified using the ADAPTAHOP algorithm. Since New Horizon is a zoom-in simulation of Horizon-AGN, galaxies close to the boundary of the zoom-in region could be polluted with low-resolution (more massive) DM particles that are initially located outside the zoom-in region. Thus, we limit our analysis to galaxies more massive than $10^{9} M_{\odot}$ in haloes with a contaminated fraction of less than $4 \% .^{10}$

Due to the nature of ADAPTAHOP, star-forming clumps inside a galaxy are identified as different galaxies; stars in these local density peaks are not taken into account as members of the main galaxy in question. To avoid such subtraction, we include all the substructures (star-forming clumps or dwarf galaxies) inside $R_{90}$ of the galaxy and remeasure $R_{90}$ to make sure that it contains $90 \%$ of the total stellar mass. Some of the galaxies in the sample are in the process of merging with massive companions. In such cases, their morphologies are likely to be highly disturbed, which makes their disk and spheroid structures unreliable. Therefore, we exclude the galaxies from the sample if they have satellite galaxies, within their $R_{90}$, with masses higher than $10 \%$ of the stellar masses of the host galaxies. The satellite galaxies in these cases are excluded from the analysis as well. Finally, we limit our sample to the galaxies with merger trees available up to at least $z=4$.

The resulting main sample consists of 144 galaxies with masses greater than $10^{9} M_{\odot}\left(24\right.$ massive galaxies with $M_{\text {stellar }} \geqslant 10^{10} M_{\odot}$ and 120 low-mass galaxies with $10^{9} M_{\odot} \leqslant M_{\text {stellar }}<10^{10} M_{\odot}$ ) at $z=0.7$, where our simulation stops. Of the 144 galaxies in the selected sample, 91 (13 massive and 78 low-mass galaxies) are free from contamination and 131 (20 massive and 111 low-mass galaxies) have a contaminated fraction below $1 \%$. The properties of these galaxies (e.g., galaxy mass function, halo mass function, star formation rate density, etc.) are in reasonable agreement with observations at the corresponding redshift considering the small sample size, which will be presented in detail in the forthcoming paper that properly introduces the New Horizon project (Y. Dubois et al. 2019, in preparation).

\section{Kinematic Decomposition}

\subsection{Definition}

Disks and spheroids are thought to be the most distinct components of a galaxy in terms of kinematics and stellar populations. In order to investigate the formation and evolution

\footnotetext{
${ }^{10}$ If a galaxy is in a subhalo, both subhalo and host halo should have lowresolution particles with number fraction lower than $4 \%$ to be part of the sample.
} 
of each component, it is first necessary to decompose galaxies into these two components. We use the circularity parameter (Abadi et al. 2003) to carry out the kinematic decomposition by quantifying the orbital property of each stellar particle in a galaxy. First, we define the galactic rotational axis (spin axis) as the direction of the net angular momentum vector using the stars within $R_{90}$. The circularity parameter is calculated based on the specific angular momentum of each stellar particle on the galactic spin axis $\left(J_{z}\right)$, normalized to that of an expected circular orbit with the same energy as the stellar particle $\left(J_{\text {cir }}(E)\right)$ :

$$
\epsilon=J_{z} / J_{\operatorname{cir}}(E) \text {. }
$$

By definition, a star on a circular orbit in the galactic plane would have a circularity parameter of 1 , while the circularity parameters of a group of randomly orbiting stars would be distributed centered around 0 .

We adopt a fixed cutoff of $\epsilon=0.5$ to determine whether a stellar particle is in the ordered (disk) or disordered (spheroidal) component. This cutoff is sufficiently small to contain stars in the thick disk component whose circularity values are typically centered around $\epsilon \sim 0.5-0.6$ (Abadi et al. 2003; Obreja et al. 2018). Some other studies have instead applied more strict cutoffs, e.g., $\epsilon=0.7$ or 0.8 (e.g., Marinacci et al. 2014; Rodriguez-Gomez et al. 2016), for extracting purely rotating thin disk components with less contamination from the spheroidal component. Our qualitative conclusion, however, does not depend critically on the choice of the cutoff. Since our main focus is the early history of galaxy evolution (down to $z=0.7$ ) where the two distinct structures begin to appear in a galaxy, we use a simple cutoff instead of adopting more sophisticated decomposition techniques (e.g., Obreja et al. 2019). Thus, we define the "spheroidal components" as all the stellar particles with disordered orbits $(\epsilon<0.5)$.

Figure 3 shows images of one of the most massive diskdominated galaxies and its two components decomposed based on the circularity parameter: disk $(\epsilon \geqslant 0.5)$ and spheroid $(\epsilon<0.5)$. The top two rows are $r$-band-weighted images (in the rest frame, without dust extinction) of the galaxy and its structures viewed face-on and edge-on respectively, while the third row shows mass-weighted images. The last row is the line-of-sight velocity map in the edge-on direction, weighted by the $r$-band flux of the stars in each bin. The $r$-band flux is calculated for every stellar particle based on stellar age and metallicity, following the stellar population synthesis models of Bruzual \& Charlot (2003). Stars with $\epsilon<0.5$ are indeed distributed smoothly as expected for the spheroidal component, whereas the disk stars with $\epsilon \geqslant 0.5$ show luminous (young) spiral-arm structures in the face-on view while looking thin in the edge-on view. In the line-of-sight velocity map, the difference between the components is also evident: stars with $\epsilon<0.5$ build a dispersion-dominated system with little net rotation because their line-of-sight velocities are canceled out, while disk stars show a clear rotation with $V_{\text {rot }} \sim 200 \mathrm{~km} \mathrm{~s}^{-1}$.

\subsection{Kinematic Morphology (D/T) - Morphological Mix in the Field Environment}

The morphology of galaxies can be approximated by the ratio of the mass of the disk components to the total stellar mass, $D / T$. Figure 4(a) shows the disk-to-total ratios of the New Horizon galaxies at $z=0.7$ as a function of stellar mass. The average values of $D / T$, represented as magenta diamonds,

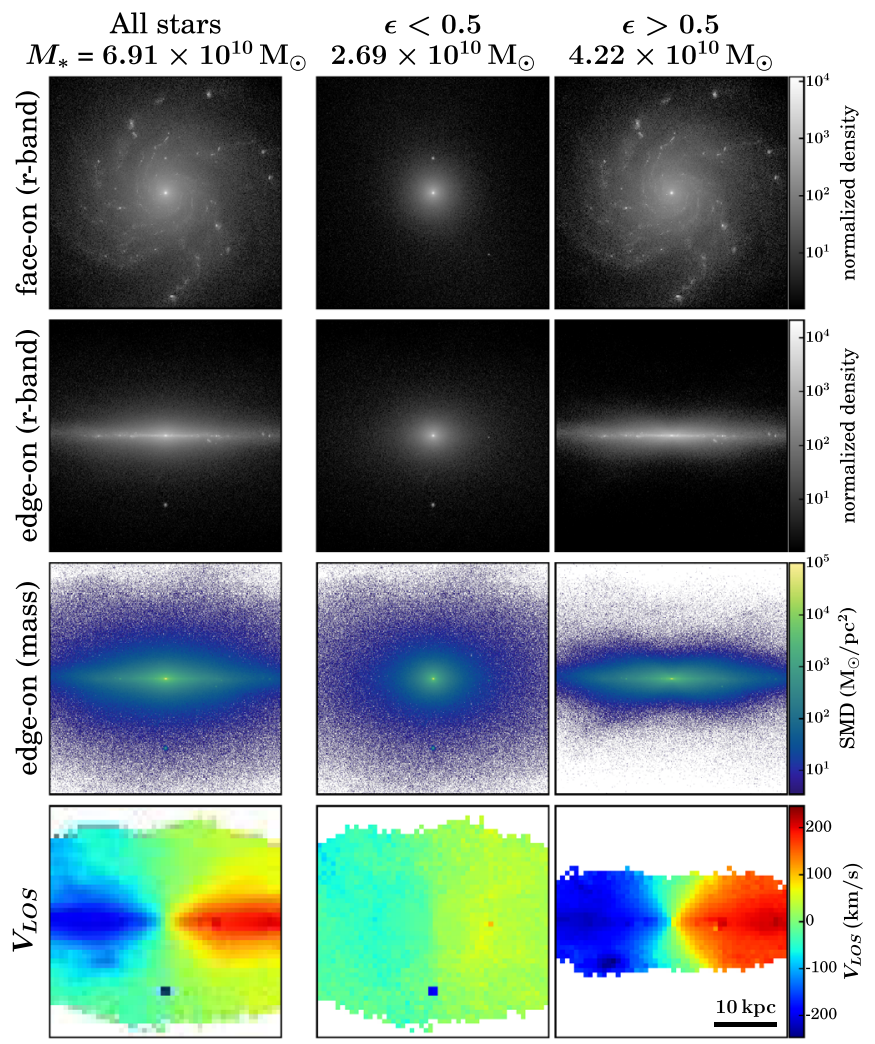

Figure 3. Images of one of the most massive disk-dominated galaxies in our sample (left) and its decomposed structures: the disk (middle) and spheroidal (right) components. The rest frame $r$-band images are shown in face-on (top row) and edge-on (second row) views. The mass distributions are shown in an edge-on view (third row), and the $r$-band flux-weighted line-of-sight velocity map in an edge-on view (fourth row). We confirm a distinction between the kinematically decomposed structures, disk and spheroid, in their visual appearance and velocity maps. The radius of each box is $R_{90}$ of the galaxy, and the velocity map is displayed on a $50 \times 50$ grid. Only pixels containing more than 100 stellar particles are shown on the map.

seem to show a slight positive trend with the stellar mass of the galaxies. As a check, we also measured $V / \sigma$ of the galaxies, the degree of rotational support. First, we define the cylindrical coordinate system with respect to galactic spin axis (z-axis) and compute the radial $\left(V_{r}\right)$, tangential $\left(V_{t}\right)$, and vertical $\left(V_{z}\right)$ components of velocity. Velocity dispersion is measured with respect to the mean value of each component (i.e., $\left.\sigma_{r, t, z}^{2}=\left\langle V_{r, t, z}^{2}\right\rangle-\left\langle V_{r, t, z}\right\rangle^{2}\right)$. Using all stellar particles in a galaxy, $V / \sigma$ is calculated from the mean rotational velocity $\left(V=\left\langle V_{t}\right\rangle\right)$ normalized by the 1D-mimicking velocity dispersion: $\sigma=\sqrt{\left(\sigma_{r}^{2}+\sigma_{t}^{2}+\sigma_{z}^{2}\right) / 3}$. As suggested in the gradual color variation of $D / T$ (representing $V / \sigma$ ), we confirm that our $D / T$ measurement correlates well with $V / \sigma .^{11}$

To examine the differences in the kinematically traced morphology more carefully, we classified the galaxies with $D / T$ larger than 0.5 as disk-dominated galaxies and those with $D / T$ smaller than 0.35 as spheroid-dominated galaxies. The rest are called intermediate galaxies. Disk-dominated galaxies make

\footnotetext{
11 Note that $V / \sigma$ here is different from observed $V / \sigma$; while we consider all the stellar particles in a galaxy in the measurement of $V / \sigma$, many of the observational studies measure $V / \sigma$ from the maps of velocity moments within the projected half-light radius (e.g., Emsellem et al. 2007). We note that $V / \sigma$ presented here is not intended for direct comparison with observations but for a sanity check on the reliability of $D / T$ measurements and to confirm the validity of the trends.
} 

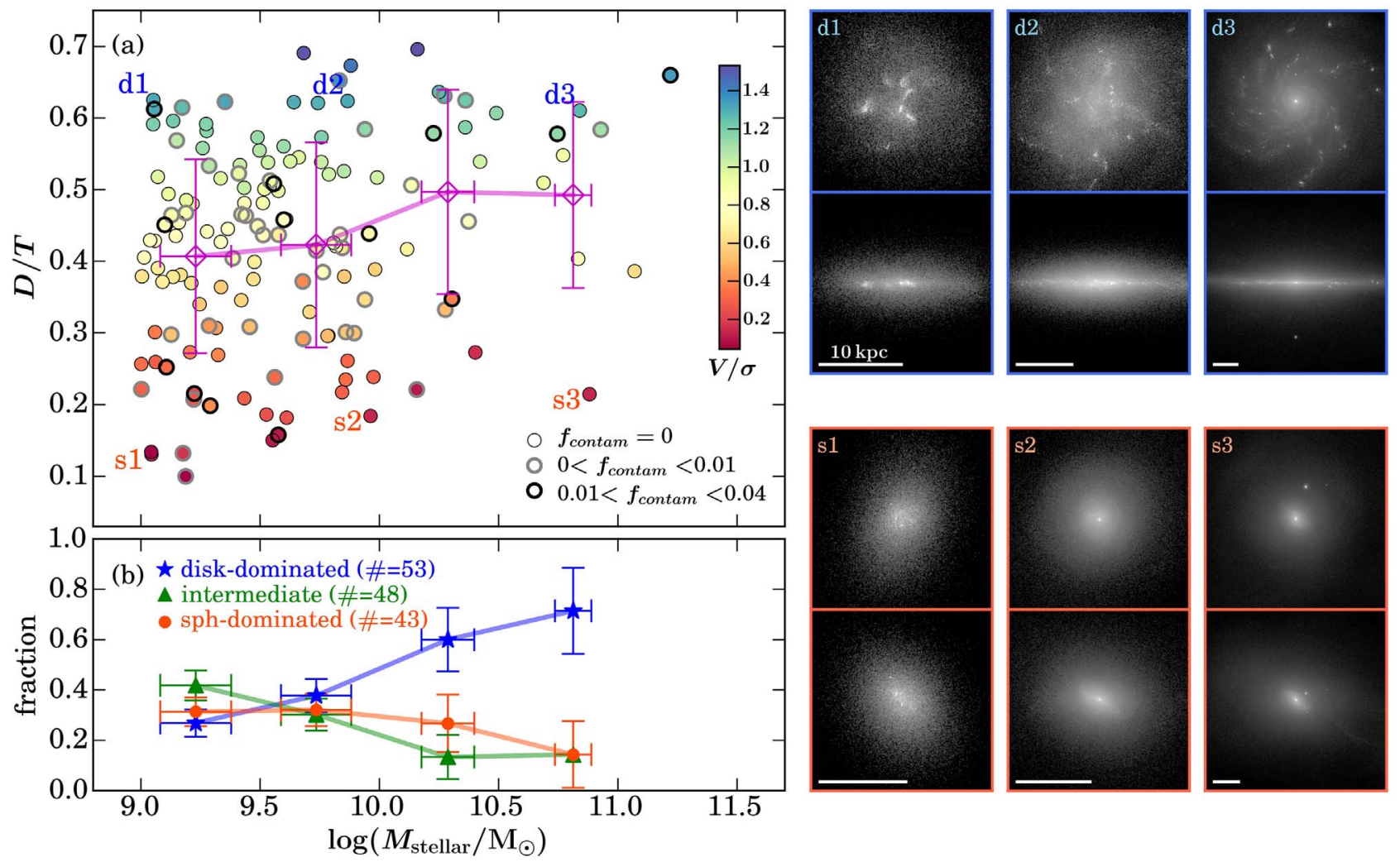

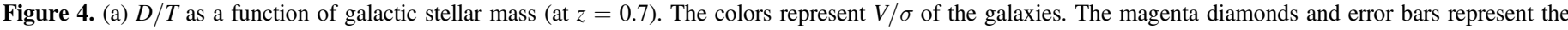

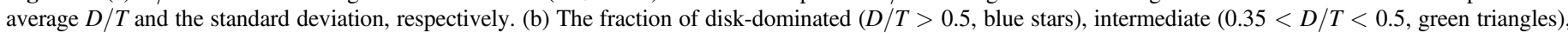

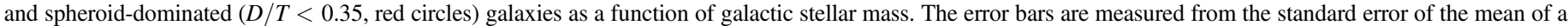

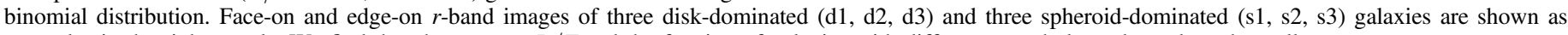
examples in the right panels. We find that the average $D / T$ and the fraction of galaxies with different morphology depend on the stellar mass.

up $37 \%$ of the sample $(53 / 144)$, whereas $30 \%$ of the sample $(43 / 144)$ is spheroid-dominated. Face-on and edge-on $r$-band images of three disk-dominated $(\mathrm{d} 1, \mathrm{~d} 2, \mathrm{~d} 3)$ and three spheroiddominated (s1, s2, s3) galaxies are shown as examples in the right panels of Figure 4. The fractions of disk-dominated (blue), intermediate (green), and spheroid-dominated (orange) galaxies in each mass bin are shown in panel (b).

It is interesting to note that, unlike the average $D / T$ (panel (a)), the fraction of disk-dominated galaxies increases rather dramatically with stellar mass. The strength of the trend, however, is somewhat sensitive to the choice of $D / T$ cuts for disk- and spheroid-dominated galaxies. The difference in the populations between disk-dominated and spheroid-dominated galaxies begins to appear at $\sim 10^{10} M_{\odot}$ and becomes more pronounced at higher masses. This morphology-mass trend is consistent with the observational studies of Kassin et al. (2012) (see also Simons et al. 2017; Johnson et al. 2018), while it may seem contradictory to what we observe in the local universe, where more massive galaxies tend to be of earlier type (Conselice 2006). This discrepancy can be explained by noting that our sample is limited to the field environment, and the simulation has not reached $z=0$.

\section{The Origin of Disk and Spheroidal Components}

Understanding the formation of the kinematic components requires tracking their stellar origins-when and where stars come from. In this section, we sorted the origins of the stellar particles in each component depending on their birthplace and orbits at birth: stars born in situ with "aligned" (corotating coplanar initial orbits) and "misaligned" orbits (counterrotating or non-coplanar initial orbits), and stars formed ex situ and later accreted. Based on this classification, we aim to address the questions raised in the introduction regarding the formation of disk and spheroidal components.

\subsection{The Origins of Stellar Particles: in situ/ex situ and Birth Orbital Properties}

The origin of stars can be quantified as a function of where they formed: in situ/ex situ. We tracked all the progenitors of the galaxy (up to at least $z=4$ ). For each galaxy of the $z=0.7$ sample, stars born in its main progenitors are considered as in situ stars, and the rest as ex situ stars. ${ }^{12}$ Figure 5 shows the distribution of stars in the same disk-dominated galaxy as in Figure 3. The top two rows show the numbers of in situ (panel (a)) and ex situ (panel (b)) stars as a function of distance. The distribution function of in situ stars is colored according to the median formation epoch of the stars at given distances. The distributions of ex situ stars assembled at $z \sim 3$ and $z \sim 1.5$ $(0.5$ Gyr window) are also added in panel (b). In the middle panels, stars are distributed in the plane of the 3D velocity and the galactocentric distance, while the bottom rows show the

\footnotetext{
$\overline{12}$ In each snapshot from (at least) $z=4$ to 0.7 , stars younger than $50 \mathrm{Myr}$ located within $R_{90}$ of the main progenitors of the galaxy in question are labeled as in situ stars, and those stars that have not been labeled at all (meaning that they are born in other progenitors and accreted later when they are older than $50 \mathrm{Myr}$ ) are considered to be ex situ stars.
} 

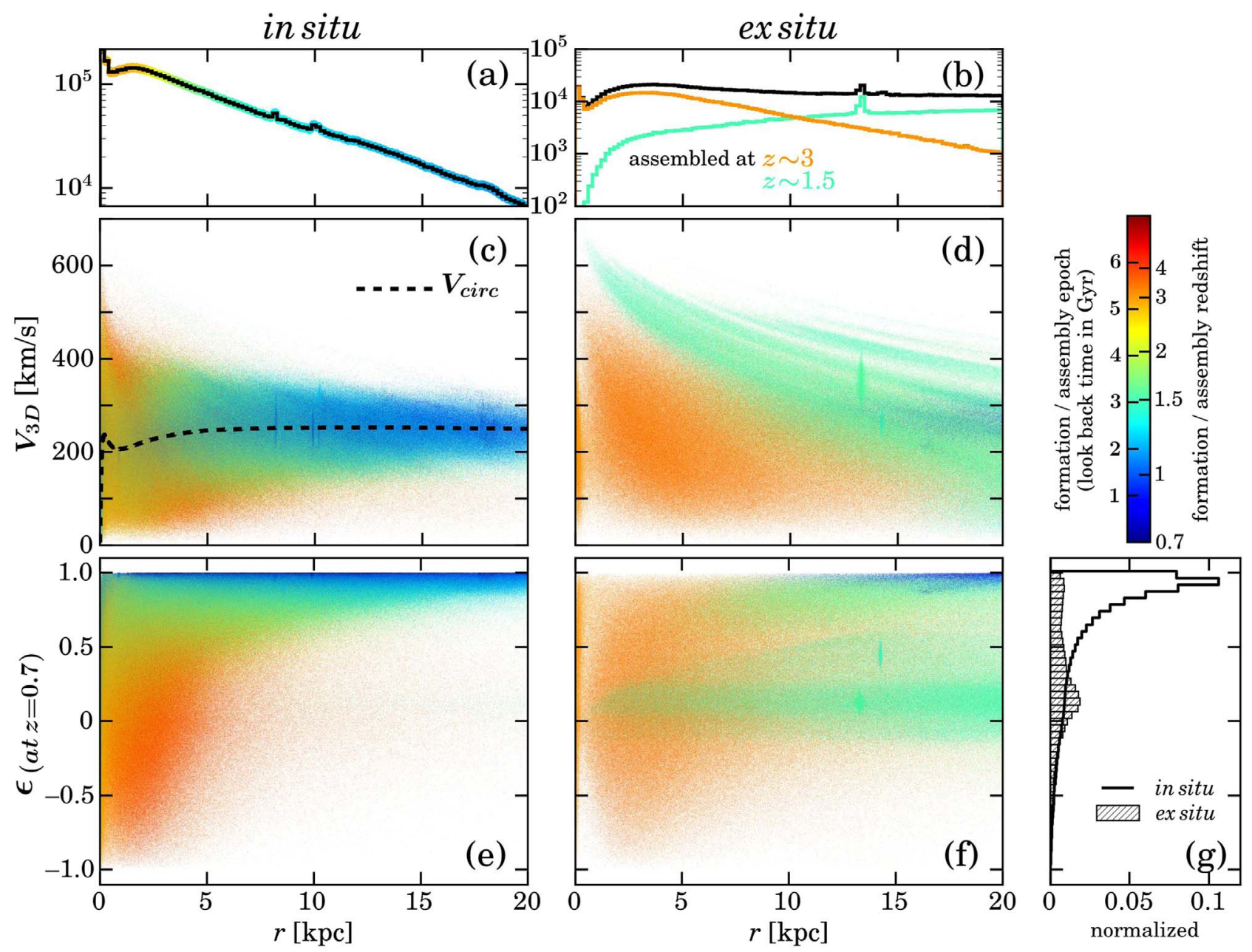

Figure 5. Distribution of the in situ and ex situ stars in a galaxy in a phase-space diagram. Top panels: the numbers of in situ and ex situ stars as a function of galactocentric distance. The distribution of in situ stars is colored by median formation epoch of the stars at given distances. Orange and cyan lines are distributions of ex situ stars assembled at $z \sim 3$ and $z \sim 1.5$ (two significant accretions through mergers), respectively. Middle panels: 3D velocity vs. the distance from the galactic center. Bottom panels: the circularity parameter vs. the distance. Each star is color-coded according to its formation (in situ) or assembly (ex situ) epoch, from red (early) to blue (recent). The rotational velocity curve of the galaxy is plotted as the dashed line in panel (c). (g) Probability distribution functions (PDFs) of the circularity parameters of in situ (unfilled) and accreted (hatched) stars normalized by the total number of stars in the galaxy. The stellar kinematics is strongly dependent on the formation/assembly epochs and origins; young stars formed in situ have ordered motions with circularity close to 1, while stars formed earlier have disordered motions with low circularity. Accreted stars have mostly disordered motions with circularity centered around 0, and their radial distributions depend on their assembly epoch.

circularity parameter versus distance. Stars in each panel are color-coded according to their formation (in situ) or assembly epoch (ex situ).

It is clear from Figure 5 that the present stellar kinematics is strongly dependent on the formation/assembly epoch and origin. The gradual color variations in panels (c) and (e) indicate that the stars formed in situ have different kinematics depending on their ages. Stars formed recently have circularity values close to 1 , following the circular rotation curve, which is represented as the dashed line in panel (c). Therefore, the majority of young stars in the galaxy are formed in the disk. Conversely, old stars formed in situ are mostly contained in the inner regions with lower circularity, many of which comprise the bulge component.

In the phase-space diagram for ex situ stars, Figures 5(d) and (f), we can identify different families of accreted stars coming from different merger events. This particular galaxy undergoes significant accretions at $z \sim 3$ (orange) and $z \sim 1.5$ (cyan) through mergers, which can be seen as different groups of accreted stars with different assembly epochs. Unlike the stars formed in situ whose circularity parameters are skewed toward $\epsilon=1$, accreted stars mostly have disordered motions with circularity parameters almost centered around $\epsilon \sim 0$ (Figure 5(g)). Moreover, the distribution of accreted stars in the phase-space plane varies with epoch of assembly, as has been addressed by Font et al. (2011). The stars from a recent accretion (cyan stars in Figures 5(d) and (f), assembled at $z \sim 1.5$ ) are distributed at large distances from the center with higher velocities, while stars accreted earlier (orange stars, assembled at $z \sim 3$ ) have settled down toward the central part of the galaxy, because later accretion carries higher angular momentum (e.g., White 1984; Kimm et al. 2011; Stewart et al. 2011).

The most visible feature in this diagram is perhaps that stars that formed earlier tend to contribute to the spheroid and to have more disordered orbits. The orbital characteristics of a star, however, are not fixed over time. The ordered orbits of disk stars can be disturbed as stars exchange angular momentum through mergers or instabilities. Therefore, stars in each component do not remain where they are born. To 

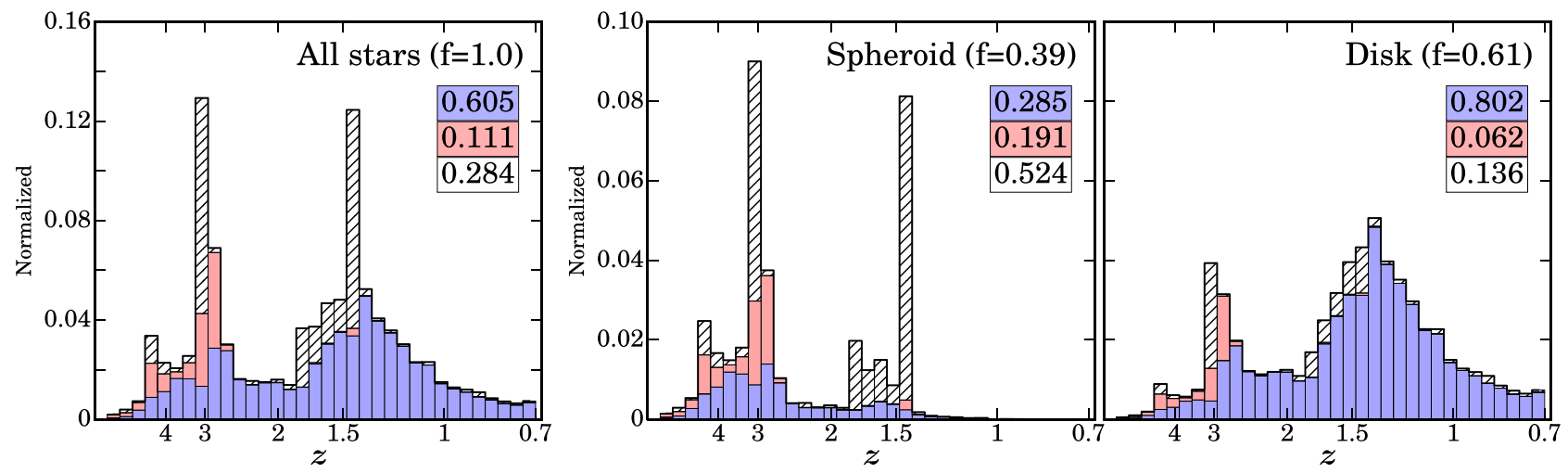

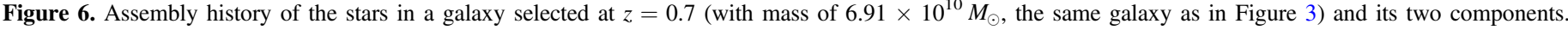

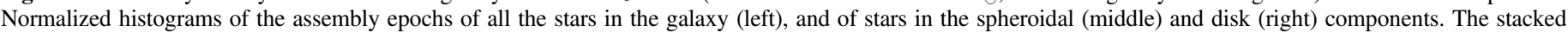

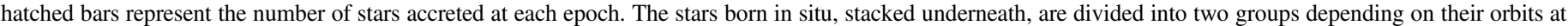
birth: aligned (blue) and misaligned (red). The fractions of each subcomponent in the spheroid and disk are shown in the upper right box.

determine which component (disk/spheroid) stars belonged to when they were born, and therefore to quantify the amount of migration from disks to spheroids, we measured the orbital properties of the in situ stars at birth. The stars born in situ were divided again based on their circularity parameter at birth. The same choice of circularity cutoff $\epsilon_{\text {birth }}=0.5$ was employed to determine whether a star was born a disk star. In other words, if the initial orbit of a star (at birth) is "aligned" with the corotating disk plane $\left(\epsilon_{\text {birth }}>0.5\right)$, then it was born a disk star. Otherwise $\left(\epsilon_{\text {birth }}<0.5\right.$, "misaligned orbits" with the corotating disk plane), it is most likely to have formed from misaligned, unsettled gas, likely during a merger.

The leftmost panel in Figure 6 shows the assembly history of the same disk-dominated galaxy as in Figure 3 with stellar mass of $6.91 \times 10^{10} M_{\odot}$. Each bin represents the number of stellar particles born in situ with aligned (blue) and misaligned (red, on top of blue) orbits and the number of stellar particles formed ex situ and later accreted by this galaxy (hatched, on top of red). The boxes in the top right corner of the panel show the fractions of these subcomponents in the galaxy. As indicated by colors in Figure 5, this galaxy experienced significant accretion events at $z \sim 3$ and $z \sim 1.5$ through mergers, resulting in the two maxima of stars accreted at these redshifts (spikes in the hatched histograms). The total ex situ mass fraction is $\sim 0.284$. At high redshifts, most stars form from misaligned and unsettled gas (red bars); later, disk stars (blue bars) start to form predominantly. The general trend of the star formation responsible for disk stars will be discussed in Section 4.2.

The two panels on the right, in the same format as the left panel, show when and how the stars constituting the spheroid (middle) and the disk (right) at $z=0.7$ are assembled into the galaxy. The spheroidal component consists of accreted stars (accounting for $52.4 \%$ of the spheroid), stars born with misaligned orbits $(19.1 \%)$, and stars initially born in the disk (initial aligned orbits) yet migrated to the spheroid as their orbits are perturbed (28.5\%). The disk component, conversely, is primarily composed of stars formed in situ with aligned orbits (more than $80 \%$, for this galaxy). The distribution of the assembly epochs of the stars in each component implies that the stars in the spheroidal component are older than the disk stars because the galaxy continuously produces young disk stars. We further calculated the median age of the spheroidal and disk components of this galaxy at $z=0.7$ and confirmed that the spheroid stars $(5.35 \mathrm{Gyr})$ are much older than the disk stars (3.01 Gyr). The qualitative assembly history of disk and spheroid and their origins (high ex situ fraction for spheroidal components, mainly for the stellar haloes given their distribution in Figure 5) agree with the results of Obreja et al. (2018).

\subsection{Formation of Disks}

As implied in Figure 6, disk stars tend to form at later epochs, while early star formation contributes to the growth of the spheroidal components. In this section, we explore when the galaxies start to predominantly form disk stars. Also, we measure how many of the stars in a galaxy are born as disk stars and investigate how this correlates with galactic stellar mass, final morphology, and accreted fraction.

\subsubsection{When Do Galaxies Start to Predominantly Form Disk Stars?- Disk-mode Star Formation}

In order to quantify the kinematic properties of newly formed young stars at each redshift, we measure the fraction of disk stars among the stars formed over a time period of $100 \mathrm{Myr}, f_{\text {disk }}^{100 \mathrm{Mrr}}$. Figure 7 (a) shows $f_{\text {disk }}^{100 \mathrm{Mr}}$ as a function of redshift for two groups of galaxies divided based on their stellar mass at $z=0.7$; the solid line with circles shows the evolution of the median $f_{\text {disk }}^{100 \mathrm{Mr}}$ for massive galaxies $\left(10<\log \left(M_{*, z=0.7} / M_{\odot}\right)<11\right)$, and the dashed line with triangles shows the evolution of lowmass galaxies $\left(9<\log \left(M_{*, z=0.7} / M_{\odot}\right)<10\right)$. Each point is the median value of $f_{\text {disk }}^{100 \mathrm{Mr}}$ measured at the corresponding redshift on the $x$-axis with a time window of $\pm 150 \mathrm{Myr}$. The colors inside the markers represent the median stellar mass of their main progenitors at the corresponding redshift.

As shown in Figure 7(a), both lines increase with time. This trend is expected for two reasons: as redshift decreases, the mergers become less frequent (e.g., Rodriguez-Gomez et al. 2015, 2016), and the angular momentum of accreted material increases (e.g., Kimm et al. 2011). The most notable feature in this trend is that $f_{\text {disk }}^{100 \mathrm{Mr}}$ increases more steeply in massive galaxies (the solid line with circles), meaning that more massive galaxies start to form disk stars at earlier epochs. This trend qualitatively agrees with the observations of Kassin et al. (2012) (see also Simons et al. 2016, 2017; Johnson et al. 2018). Based on gas kinematics, they measured the fraction of galaxies with settled disks (probed by $V / \sigma$ ) and found that the fraction increases with stellar mass and time. Recent numerical studies have also reproduced this trend. For example, El-Badry et al. (2018) used 24 FIRE galaxies with stellar masses of 

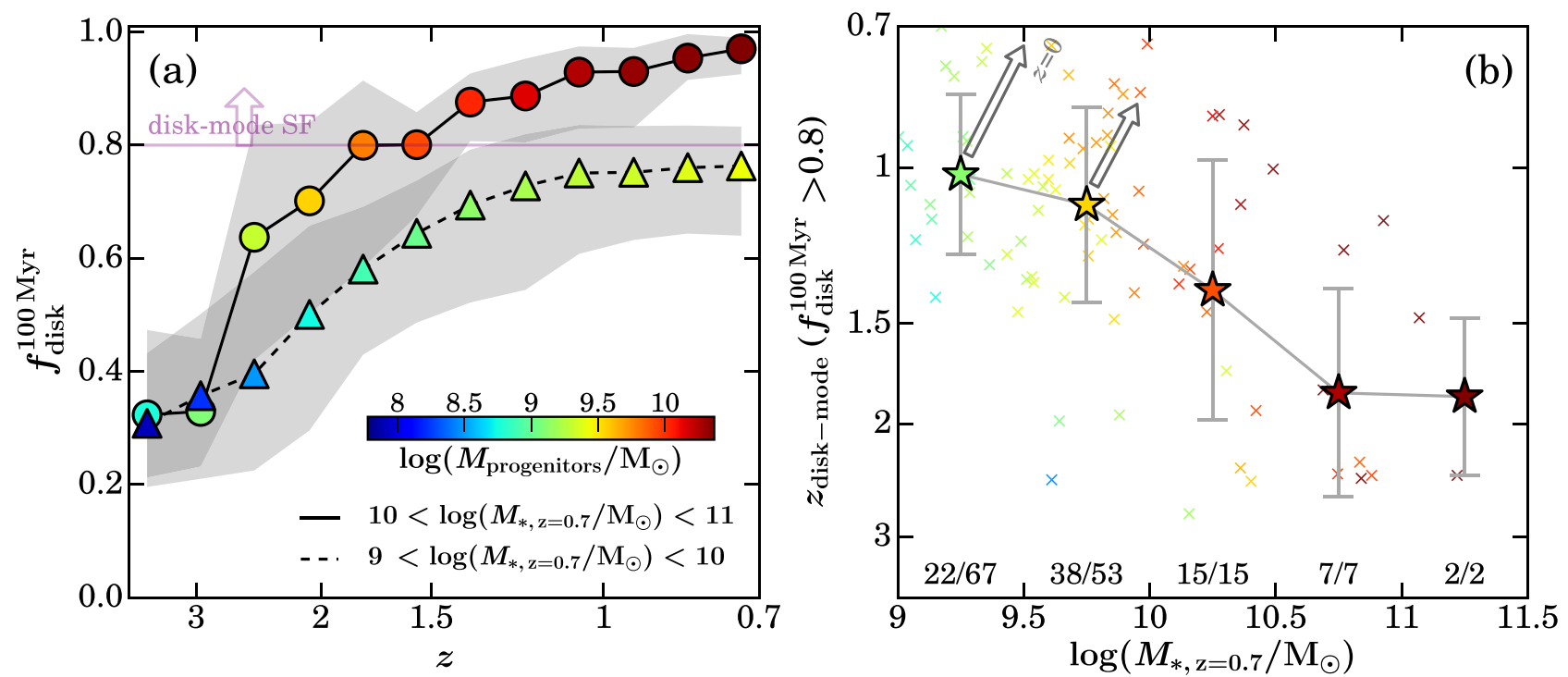

Figure 7. (a) The fraction of disk stars among the stars formed over a time period of $100 \mathrm{Myr}\left(f_{\mathrm{disk}}^{100 \mathrm{Myr}}\right)$ as a function of redshift. The solid line with circles shows the evolution of median $f_{\text {disk }}^{100}$ myr for massive galaxies $\left(10<\log \left(M_{*, z=0.7} / M_{\odot}\right)<11\right)$, and the dashed line with triangles shows the evolution of low-mass galaxies $\left(9<\log \left(M_{*, z=0.7} / M_{\odot}\right)<10\right)$. The two groups of galaxies are divided according to their stellar mass at $z=0.7$, and the colors inside the markers represent the median stellar mass of their main progenitors at the corresponding redshift. The gray shadings represent the 30th and 70th percentiles. (b) $z_{\text {disk-mode }}$, the epoch at which galaxies begin to form stars in "disk-mode" (i.e., $f_{\text {disk }}^{100 \mathrm{Mr}}>0.8$ for the last $300 \mathrm{Myr}$ ), as a function of stellar mass of galaxies. Each star represents the epoch averaged over the galaxies in the corresponding mass bin, and the error bars show the standard deviations. The colors inside the stars are again coded by the average stellar mass of the progenitors at $z_{\text {disk-mode }}$ (i.e., $M_{\text {disk-mode }}$ ). The bottom numbers indicate the number of galaxies that have ever undergone disk-mode star formation by $z=0.7$ out of the total number of galaxies in the mass bin. Thus, only a small fraction of the low-mass galaxies are taken into account in the measurement of $z_{\text {disk-mode. If we }}$ assume that those galaxies form stars in disk mode after $z \sim 0.7$ so that we count all of the low-mass galaxies in the calculation, the estimated $z_{\text {disk-mode }}$ for the two lowest mass bins will likely to move up and rightward as marked by arrows (scale and direction are arbitrary). We find that more massive galaxies (e.g., $\left.M_{*, z=0.7}>10^{10} M_{\odot}\right)$ start to form disk stars from earlier epochs $(z \sim 1-2)$.

$6<\log \left(M_{*} / M_{\odot}\right)<11$ and found a mass dependence of the formation of rotationally supported gas disks. In addition, Pillepich et al. (2019) have shown a similar trend-that more massive galaxies have thinner disks, in the sense of better defined morphological structure and kinematics, than less massive ones. This disk settling issue will be discussed in more detail in a separate paper (Y. Dubois et al. 2019, in preparation).

Another noticeable feature of Figure 7(a) is that high-redshift galaxies with settled disks (progenitors of the massive galaxies at $z=0.7$ ) are heavier than galaxies whose disks settled more recently; the colors inside the markers, representing the median progenitor mass at the corresponding redshift, are redder to the left at fixed $f_{\text {disk }}^{100 \mathrm{Mrr}}$ (in the horizontal direction). In order to investigate the mass associated with disk settling more carefully, we define disk-mode star formation as when $f_{\text {disk }}^{100 \mathrm{Myr}}$ is higher than $0.8^{13}$ for the last $300 \mathrm{Myr}$. We measure "when" galaxies "begin" this disk-mode star formation (diskmode epoch, $z_{\text {disk-mode }}$ ). Figure $7(\mathrm{~b})$ shows $z_{\text {disk-mode }}$ as a function of stellar mass of galaxies at $z=0.7$. Each star represents $z_{\text {disk-mode }}$ averaged over the galaxies in the corresponding mass bin, and the color is coded by $M_{\text {disk-mode, }}$, the median mass of the progenitors at $z_{\text {disk-mode }}$.

The trend in Figure 7(b) clearly shows an anticorrelation between galactic stellar mass and disk-mode epoch; massive galaxies $\left(10<\log \left(M_{*, z=0.7} / M_{\odot}\right)<11\right)$ start to form disks from $z \sim 1-2$ (e.g., Simons et al. 2016; Hung et al. 2019), when their

\footnotetext{
13 The resulting qualitative trend does not depend sensitively on the choice of cutoff for disk-mode star formation. However, we take the high cutoff of $f_{\text {disk }}^{100 \mathrm{Mr}}=0.8$ to prevent any contamination that might be caused by our simple way of distinguishing disk-born stars $\left(\epsilon_{\mathrm{birth}}>0.5\right)$.
}

mass become $\sim 10^{10} M_{\odot}$ (e.g., Clauwens et al. 2018; Trayford et al. 2019), while low-mass galaxies $\left(9<\log \left(M_{*, z=0.7} / M_{\odot}\right)<10\right)$ do after $z \sim 1$. In addition, $M_{\text {disk-mode }}$ (the colors inside the markers) seems to mildly decrease with decreasing $z_{\text {disk-mode }}$, which can be interpreted as a result of decreasing violence of the environments at lower redshifts. At higher redshifts, where frequent star-forming activity, mergers, and gas accretion trigger gas turbulence (e.g., Genel et al. 2012; Turner et al. 2017), only massive galaxies in deep gravitational potential wells are able to form a majority of stars in "disk mode." As redshift decreases, the environments become less violent, and galaxies with lower mass begin diskmode star formation.

One thing we also need to consider is that not all low-mass galaxies are taken into account in the measurement of this diskmode epoch. The numbers at the bottom of Figure 7(b) indicate the number of galaxies that have ever undergone disk-mode star formation by $z=0.7$ out of the whole galaxy sample in the mass bin. For instance, only around a third (22/67) of the galaxies with masses between $10^{9}$ and $10^{9.5} M_{\odot}$ appear in this figure (each is marked as " $\times$ "), and the rest of them have never formed stars in "disk mode". Therefore, assuming that those galaxies will begin disk-mode star formation at some point between $z=0.7$ and $z=0$, eventually in the local universe, the two points in the lowest mass bins will be likely higher if measured at $z=0.0$, while the other points may not move much. The galaxies in the two lowest mass bins will probably grow in mass as well; and thus the two data points with the lowest mass in this diagram will likely move up and rightward as marked by arrows, while the exact direction and magnitude are unclear at the moment. As a result, we expect a steeper trend between the stellar mass and $z_{\text {disk-mode }}$ at $z=0$ than what appears here at $z=0.7$. 


\subsubsection{How Many Stars Are Born as Disk Stars in Total?}

The kinematic morphology, i.e., mass ratio of (corotating) disk to total, in this study is determined using all the stellar particles in a galaxy. Therefore, even if a galaxy has recently started disk-mode star formation, it cannot be classified as a "kinematically" disk-dominated galaxy, because the newly formed stars cannot yet outnumber the pre-existing stars, which have disordered motions. As the global star formation rate peaks near $z \sim 2$ (e.g., Hopkins \& Beacom 2006), the overall number of disk-born stars should be driven by the stars formed at these epochs. In this context, we measure the fraction of disk stars at birth $\left(f_{\text {disk }}^{\text {at birth }}\right)$ to quantify the independent effects of star formation in developing disks and spheroids, excluding the effects of migration between the components and merger accretions. The total disk fraction at birth ${ }^{14}$ is defined as the total number of stars born as (corotating) disk stars among the stars formed in situ, which is shown as a function of the stellar mass of the galaxies in Figure 8(b):

$$
f_{\text {disk }}^{\text {at birth }}=\frac{\text { in situ born stars with } \epsilon_{\text {birth }}>0.5}{\text { total number of in situ born stars }} .
$$

Each galaxy is color-coded according to its $D / T$ at $z=0.7$, and the size of the circle indicates the fraction of accreted (ex situ) stars.

In Figure 8(b), we found that the fraction of disk stars at birth increases with mass (see the magenta line). This is because, as shown in Figure 7, the heavier the galaxy, the earlier it starts disk-mode star formation. Therefore, most stars in massive galaxies $\left(>10^{10} M_{\odot}\right)$ are born as disk stars $\left(f_{\text {disk }}^{\text {at birth }} \sim 0.76\right.$, on average), which is in agreement with the results of the numerical simulation by Garrison-Kimmel et al. (2018). They found that $60 \%-90 \%$ of the stars in MW-mass galaxies at $z=0$ are born as disk stars. This mass dependence of the initial fraction of disk stars at birth is blurred by migration between the components and accretion, resulting in the weaker mass dependence on $D / T$ shown in Figure 4(a).

Another notable point is that $f_{\text {disk }}^{\text {at birth }}$ is also related to $f_{\text {accr }}$, as can be seen in the two contours in Figure 8(b). Galaxies with $f_{\text {accr }}>0.2$ (solid contour) lie diagonally across the $f_{\text {disk }}^{\text {at birth }}$ $-M_{\text {stellar }}$ plane, which is thought to be the result of the following two effects: (i) more massive galaxies have higher accretion (e.g., Oser et al. 2010; Lee \& Yi 2017) and (ii) galaxies with higher accretion have lower $f_{\text {disk }}^{\text {at birth }}$ at fixed mass (specifically, in the mass range $10^{9}-10^{10} M_{\odot}$ ). In regard to (ii), it seems that mergers or fly-bys can boost gas turbulence in galaxies, making galaxies less likely to form stars in an orderly fashion (lowering $\left.f_{\text {disk }}^{\text {at birth }}\right)$. The formation of non-disk stars $\left(\epsilon_{\text {birth }}<0.5\right)$ can be boosted by the central starburst (e.g., Hernquist 1989).

Based on the relationship between $M_{\text {stellar }}, f_{\text {disk }}^{\text {at birth }}$, and $f_{\text {accr }}$, we can understand the different morphology of galaxies as follows. The blue and orange lines in Figure 8 represent the median $f_{\text {accr }}$ (panel (a)) and $f_{\text {disk }}^{\text {at birth }}$ (panel (b)) as a function of stellar mass for disk-dominated $(D / T>0.5)$ and spheroid-dominated $(D / T<0.35)$ galaxies, respectively. Disk-dominated galaxies, on

\footnotetext{
${ }^{14}$ Note that we only considered stars with corotating and coplanar orbits as "disk" component when measuring $D / T$ (mass ratio of stars with $\epsilon>0.5$ ) and $f_{\text {disk }}^{\text {at birth }}\left(\epsilon_{\text {birth }}>0.5\right)$. Thus, due to our definition, stars formed in the counterrotating disks, whose orbits are still coplanar but counter-rotating (e.g., $\left.\epsilon_{\text {birth }}<-0.5\right)$, have "misaligned" initial orbits, and do not contribute to increasing $f_{\text {disk }}^{\text {at birth }}$. We will discuss the development of counter-rotating disks from the gas infall misaligned with the existing corotating disk plane after mergers in more detail in Section 4.4.
}
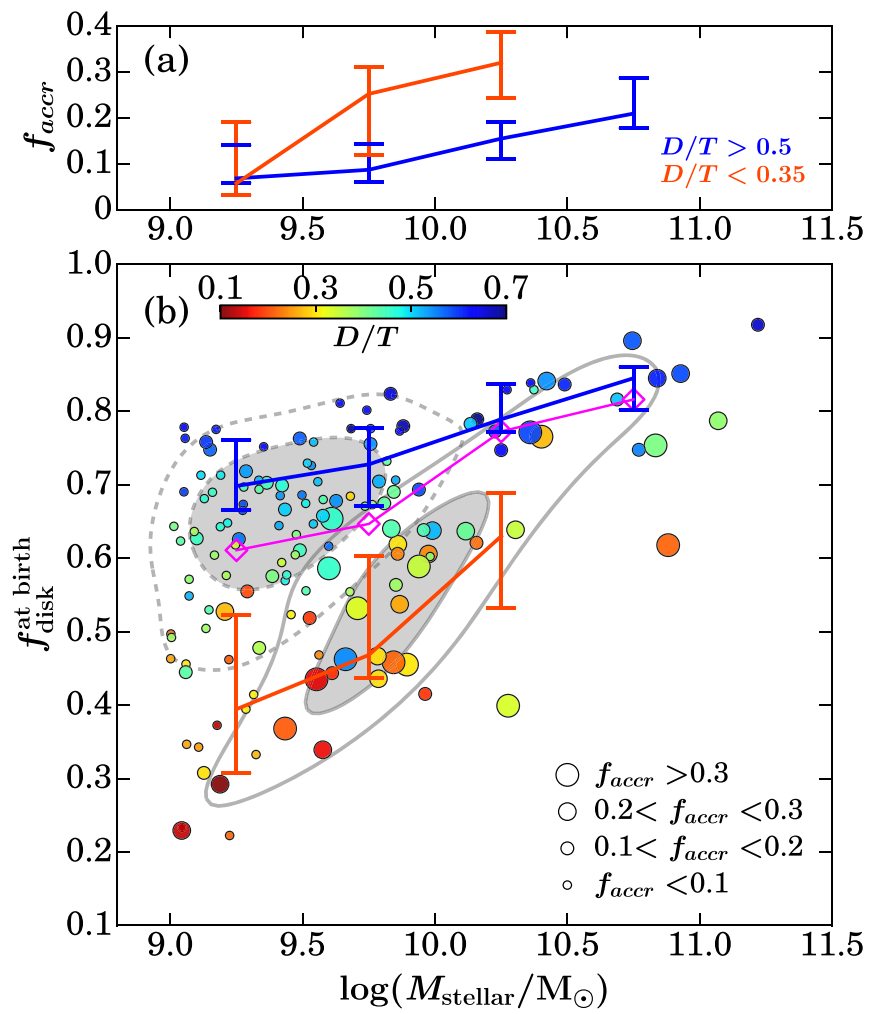

Figure 8. (a) Median fraction of accreted (ex situ) stars in the disk-dominated (blue line) and spheroid-dominated (orange line) galaxies in each stellar mass bin. (b) The fraction of disk stars at birth $\left(f_{\text {disk }}^{\text {at birth }}\right)$ as a function of galactic stellar mass. The median $f_{\text {disk }}^{\text {at birth }}$ in each mass bin is shown as the magenta line with diamonds. Each galaxy is colored according to $D / T$ at $z=0.7$, and the size of the circle indicates the fraction of accreted stars. In other words, a larger circle implies that a galaxy had more significant mergers (in the sense of either number or impact) than a galaxy plotted as a small circle. The solid and dashed contours show (shaded: $0.5 \sigma$, unshaded: $1 \sigma$ ) the distribution of galaxies with $f_{\text {accr }}$ higher and lower than 0.2 , respectively, in the plane of $M_{\text {stellar }}-f_{\text {disk }}^{\text {at birth }}$. The blue and orange lines represent the median $f_{\text {disk birth }}^{\text {at }}$ for disk-dominated $(D / T>0.5)$ and spheroid-dominated $(D / T<0.35)$ galaxies in each mass bin. All the error bars in this figure represent the 20th and 80th percentiles. $f_{\text {disk }}^{\text {at birt }}$ is strongly dependent on the stellar mass and accretion, and it is crucial in determining the final kinematic morphology.

average, have higher $f_{\text {disk }}^{\text {at birth }}$ and lower $f_{\text {accr }}$ than spheroiddominated galaxies at fixed stellar mass. This means that they have started to form disk stars from earlier epochs (thus, high $f_{\text {disk }}^{\text {at birth }}$ ) and experienced fewer violent events that can destroy their disks (hence, high $D / T$ at $z=0.7$, the final epoch).

The majority of stars in the spheroid-dominated galaxies ( $D / T<0.35$, orange lines), on the other hand, are originally formed with misaligned orbits (low $f_{\text {disk }}^{\text {at birth }}$ ). This appears to be more pronounced in the lower-mass galaxies $\left(<10^{10} M_{\odot}\right)$ that have not developed disks until $z=0.7$ (see also Figure 7). Massive spheroid-dominated galaxies $\left(>10^{10} M_{\odot}\right)$, conversely, form a higher fraction of disk stars (although still lower than disk-dominated galaxies with similar masses). Given their distinctly high $f_{\text {accr }}$, it can be assumed that mergers have made a significant contribution to the development of their spheroids.

\subsection{Growth of Spheroids}

Spheroids are believed to grow through several internal and external processes including early star formation, disk instability, and hierarchical merging. Indeed, Trayford et al. (2019) have 
^ - Spheroid stars born in situ aligned

- -- - Spheroid stars born in situ misaligned

......... Spheroid stars born ex situ
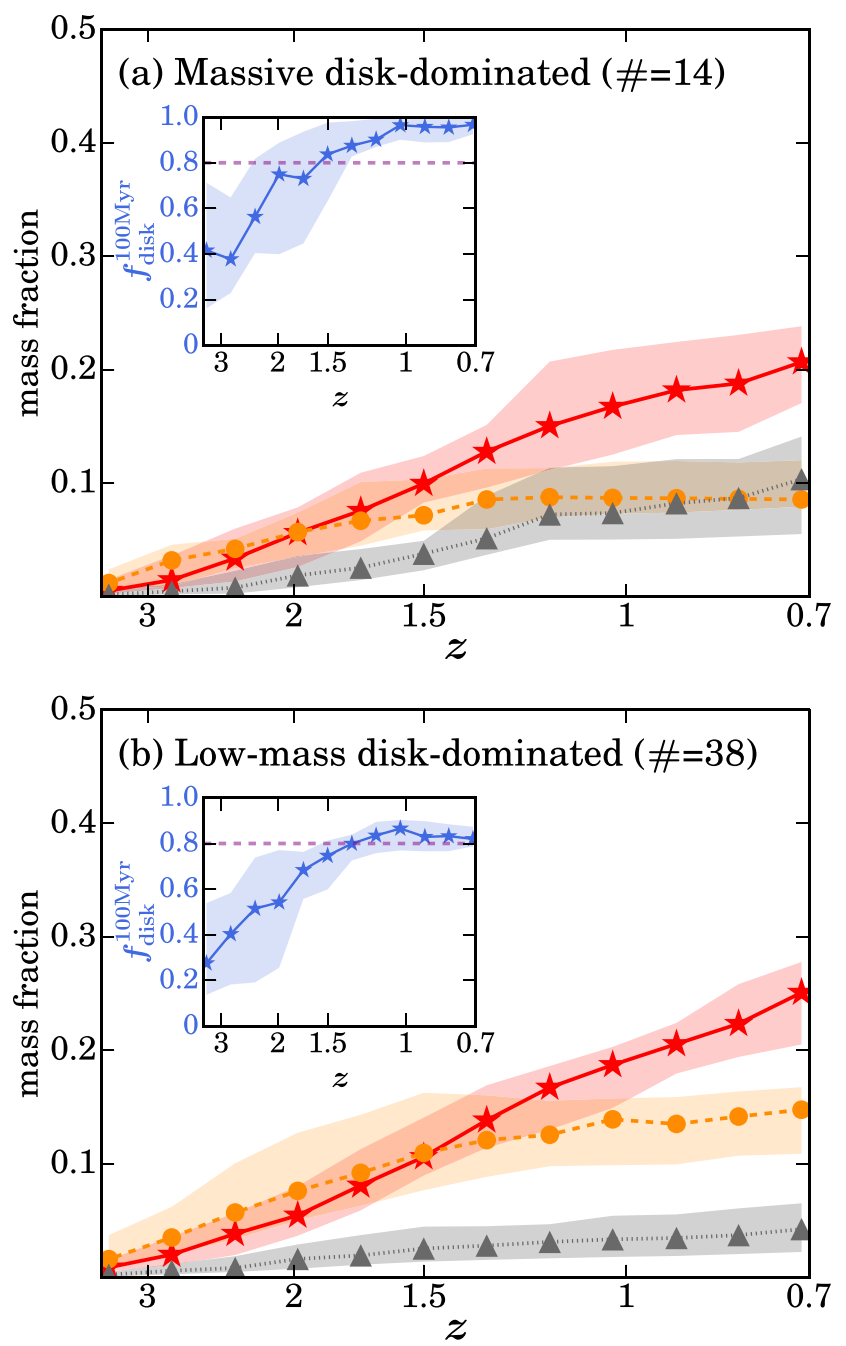
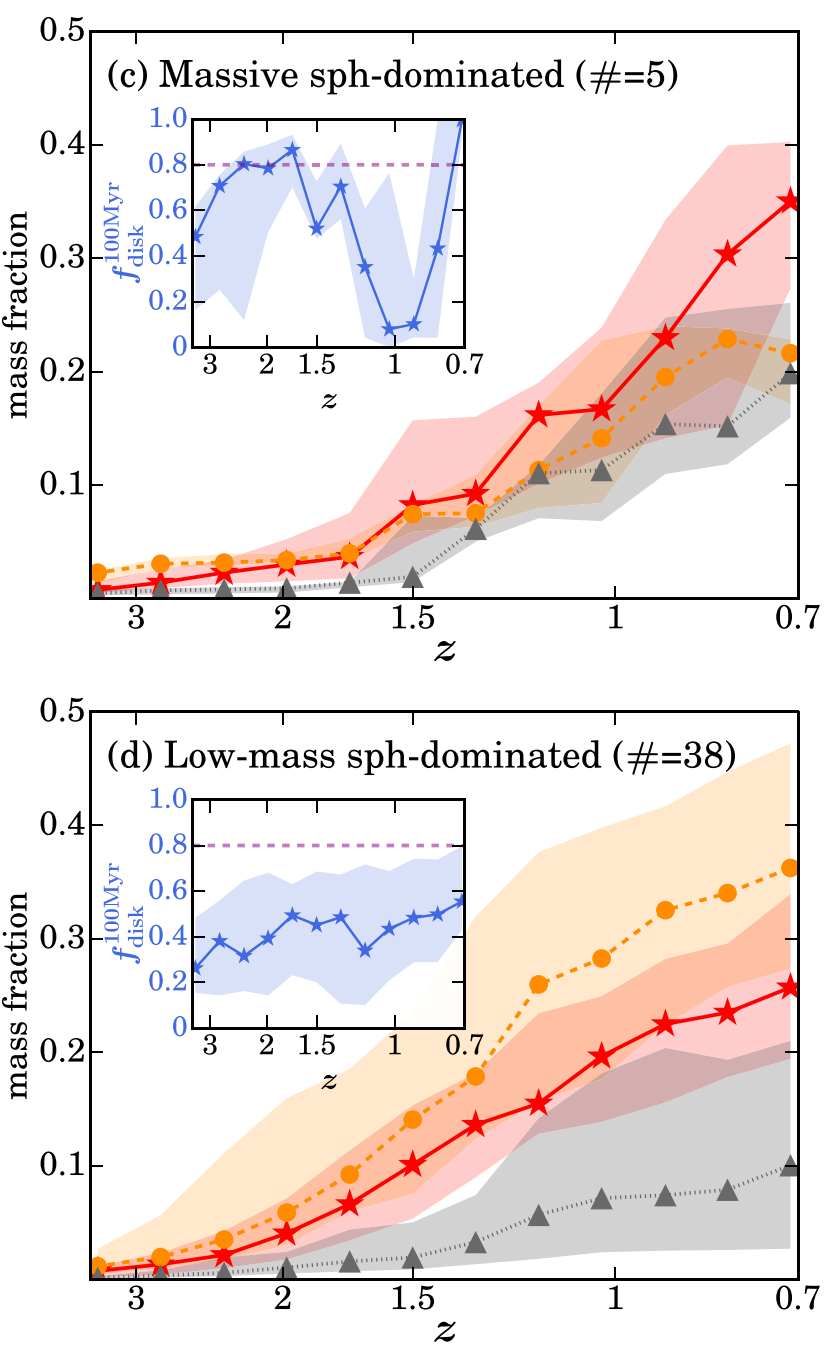

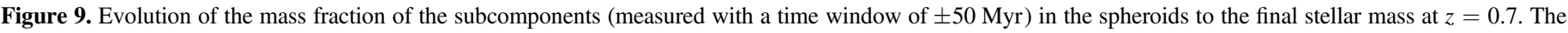

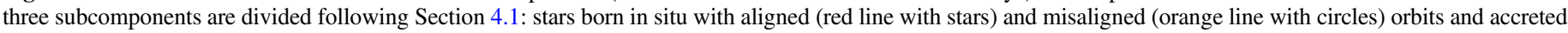

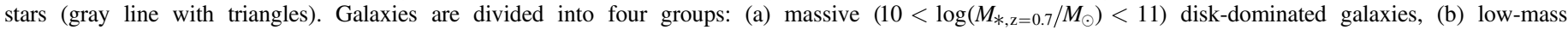

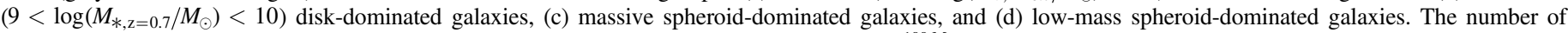

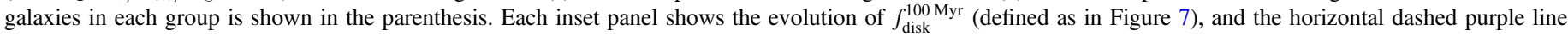

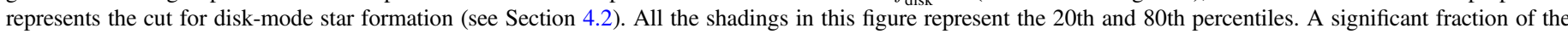

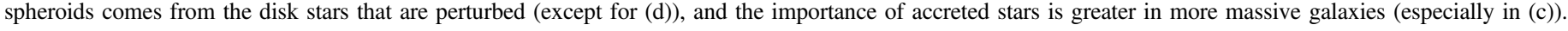

pointed out that while stars formed at high redshifts generally contribute to the spheroidal components, transformational processes of disk stars formed at lower redshifts are also necessary to build the spheroid. Thus, quantifying the different origins of spheroid stars can provide hints about the importance of each formation process. As described in Section 4.1, we divided the origin of the stars according to their birthplace and birth orbital properties: disk stars born in situ (aligned birth orbits), spheroid stars born in situ (misaligned birth orbits), and accreted stars. Figure 9 shows the evolution of the mass fraction of each subcomponent in the spheroidal components to the total stellar mass at $z=0.7$. Galaxies are divided into four groups by their final mass and morphology: (a) massive $(10<$ $\left.\log \left(M_{*, z=0.7} / M_{\odot}\right)<11\right)$ disk-dominated galaxies, (b) lowmass $\left(9<\log \left(M_{*, z=0.7} / M_{\odot}\right)<10\right)$ disk-dominated galaxies, (c) massive spheroid-dominated galaxies, and (d) low-mass spheroid-dominated galaxies. Each inset panel shows the evolution of $f_{\text {disk }}^{100 \mathrm{Mr}}$ (defined as in Section 4.2.1) for the galaxies in that group.

In the disk-dominated galaxies (panels (a) and (b)), the growth of the spheroidal components is primarily driven by the increase in perturbed disk stars (red solid lines). As the inset panel shows, most of the stars formed at lower redshift are disk stars ( $f_{\text {disk }}^{100 \mathrm{Mr}}>0.8$, disk-mode star formation); therefore, their migration into the spheroidal components is the leading contributor to the growth of the spheroids, while the subcomponent consisting of the stars born with misaligned orbits (non-disk stars, orange dashed lines) does not grow much after $z \sim 1.5$. This trend of increasing fraction of the spheroid stars that migrated from the disks agrees with the results from 
Zolotov et al. (2015); their simulation, which reached $z=1$, suggests that the fraction of these stars in spheroids is growing from $10 \%$ at $z=5$ to $30 \%$ at $z=1$ when averaged over 26 galaxies with masses higher than $10^{10} M_{\odot}$, although they used a different circularity and spatial cut to extract the spheroidal (bulge) components. As discussed in Section 4.2.2, accreted stars (gray dotted lines) seem to be more important in the more massive galaxies.

In the spheroid-dominated galaxies, each fraction of subcomponent at $z=0.7$ is almost twice as high as that of the disk-dominated galaxies with similar mass; for example, the spheroids with accreted stars (gray dotted line) account for $\sim 20 \%$ of the total stellar mass in massive spheroid-dominated galaxies (panel (c)), whereas their contribution to the total stellar mass is only $\sim 10 \%$ in massive disk-dominated galaxies (panel (a)). As Figure 8(a) shows, spheroid-dominated galaxies have a higher accreted fraction $\left(f_{\text {accr }}\right)$ than disk-dominated galaxies at fixed stellar mass, and most of the accreted stars are more likely to build up the spheroidal components.

In particular, the spheroid-dominated galaxies have a larger contribution from non-disk stars (born with misaligned orbits, orange dashed line) than disk-dominated galaxies. Low-mass spheroid-dominated galaxies (panel (d)) have not formed stars in "disk mode" at all (i.e., $f_{\text {disk }}^{100 \mathrm{Mr}}<0.8$, throughout the history until $z \sim 0.7$ as shown in the inset panel), and their spheroids grow mainly from the stars formed with misaligned orbits. On the other hand, massive spheroid-dominated galaxies (panel (c)) form stars in disk mode already at $z \sim 2$ (inset panel). The mass of their spheroidal components, however, dramatically increases after $z \sim 1.5$, along with the sudden increase in accreted stars and the subsequent decrease in $f_{\text {disk }}^{100 \mathrm{Mrr}}$ (see Section 4.2.2); the formation of stars with aligned orbits (disk stars) seems to be suppressed due to merger-boosted gas turbulence. Furthermore, disk stars dramatically migrate to the spheroidal components (red solid line), which is very likely linked to the morphological transformation induced by mergers.

\subsection{Misaligned Gas Stream- Counter-rotating Structures}

During mergers or in the early times of galaxy formation, stars form primarily from unsettled gas. Following Section 4.1, we identified these stars, which are born in situ with misaligned orbits, using their circularity at birth, and found that they generally contribute to the spheroidal components. Indeed, many of the stars in spheroid-dominated galaxies at $z=0.7$ have misaligned orbits at birth (leading to lower $f_{\text {disk }}^{\text {at birth in }}$ Figure 8). However, galaxies sometimes develop counterrotating disks due to the gas infalling in a direction misaligned with the existing corotating disk plane (i.e., sign reversal in the angular momentum of the accreted gas). This has been found in several numerical studies (Scannapieco et al. 2009; Zolotov et al. 2015; Clauwens et al. 2018; Garrison-Kimmel et al. 2018), while only a few galaxies have been observed to have such structures (e.g., Johnston et al. 2013). Because the formation of a gaseous disk should precede the development of a second, counter-rotating disk, we examined the evolution of massive galaxies $\left(>10^{10} M_{\odot}\right)$ to find such structures. Of the 24 massive galaxies in our sample, three have developed counterrotating components. In our sample, all these three galaxies build counter-rotating structures after mergers. We show the evolution of one of the galaxies in Figure 10.
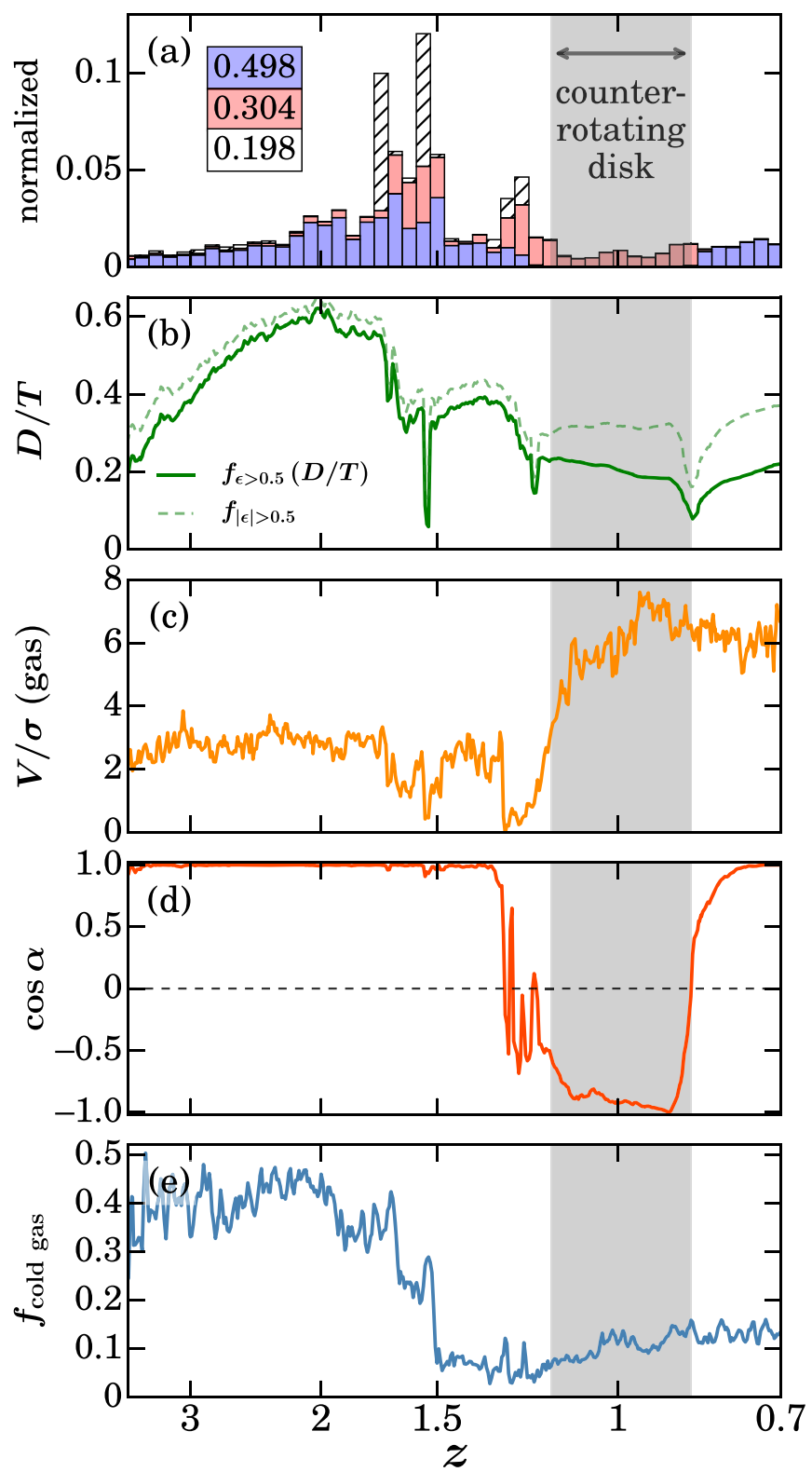

Figure 10. Evolution of a galaxy developing a counter-rotating disk. (a) The normalized stacked histogram of the assembly epochs of all stars in the galaxy, color-coded according to the different origins of the stars in the same way as in Figure 6 (blue: stars formed in situ with $\epsilon_{\text {birth }}>0.5$; red: stars formed in situ with $\epsilon_{\text {birth }}<0.5$; hatched: stars formed ex situ). The fraction of each subcomponent is shown in the box. (b) The evolution of $D / T$ (corotating disk fraction, $\left.f_{\epsilon}>0.5\right)$ as a function of the redshift. The dashed line shows the mass ratio of the stars with $|\epsilon|>0.5$ (coplanar orbits including both corotation and counter-rotation). (c) $V / \sigma$ of the cold gas inside the galaxy, measured with respect to the spin axis of the gas. (d) Cosine of the angle between the rotational axes of the gas and stars $(\cos \alpha)$. (e) The fraction of cold gas $\left(f_{\text {cold gas }} \equiv M_{\text {cold gas }} /\left(M_{\text {stellar }}+M_{\text {cold gas }}\right)\right)$ as a function of the redshift. The gray shade represents the duration of the counter-rotating disk $(V / \sigma>3$ and $\cos \alpha<0)$.

Figure 10 shows the evolution of one of the galaxies that developed a counter-rotating disk of gas (and/or recent stars). From top to bottom the panels show (a) the assembly history of stars with different origins, classified as in Section 4.1, (b) the evolution of $D / T$, (c) $V / \sigma$ (gas), (d) the cosine of the angle between the gas and stellar rotational axes $(\cos \alpha)$, and (e) the fraction of cold gas $\left(f_{\text {cold gas }} \equiv M_{\text {cold gas }} /\left(M_{\text {stellar }}+M_{\text {cold gas }}\right)\right)$ 
as a function of redshift. The gas kinematics is also measured using the cold gas $\left(n_{\mathrm{H}}>10 \mathrm{~cm}^{-3}\right.$ and $\left.T<2 \times 10^{4} \mathrm{~K}\right)$ within $R_{90}$. To identify a misaligned disk, $V / \sigma$ of the gas was measured with respect to the gas rotational axis (direction of net angular momentum), not with respect to the spin axis of the stars. We define that a galaxy develops a counter-rotating structure when $V / \sigma$ of the galactic gas is higher than 3 while the angle $(\alpha)$ between the spin axes of gas and stars is greater than $90^{\circ}(\cos \alpha<0)$.

As shown in Figure 10, the galaxy encountered a satellite galaxy at $z \sim 1.56$ (with a mass ratio of $\sim 1: 4$ ), leading to a significant accretion of satellite stars (hatched bars in panel (a)). The merger ended at $z \sim 1.24$, and since then the spin axis of the gas has started to deviate. While the gas has a high rotational support (high $V / \sigma$ in panel (c)), its spin axis is nearly opposite to the stellar rotational axis ( $\cos \alpha \sim-1$ in panel (d)). Therefore, the stars formed in this counter-rotating disk also have negative circularity at birth (e.g., $\epsilon_{\text {birth }} \sim-1$, represented as red bars in panel (a)), thus reducing the fraction of corotating disk, $D / T$ (i.e., $f_{\epsilon}>0.5$ ) in panel (b). In panel (b), we also add the mass ratio of the stars with $|\epsilon|>0.5$, coplanar orbits including both corotation and counter-rotation, as the green dashed line. For most of the time (until $z \sim 1.24$ ), $f_{\epsilon>0.5}$ and $f_{|\epsilon|>0.5}$ do not show much difference, though $f_{|\epsilon|>0.5}$ is systemically higher by definition. When there is a sign reversal of the angular momentum of the infalling gas (during the gray shaded period), however, the counter-rotating disk $(\epsilon<-0.5)$ grows, while the existing corotating disk $(\epsilon>0.5)$ shrinks; therefore $f_{|\epsilon|>0.5}$ remains almost constant.

This counter-rotating disk lasts more than a gigayear until $z \sim 0.85$, when the two spin axes finally align. The alignment of the spin axes is caused by the reversal of the stellar rotational axis due to the two combined effects: (i) the kinematics of the original system was dominated by disordered motion (low $D / T$ at $z \sim 1.2$ ); therefore, the total angular momentum of the system was small. (ii) Meanwhile, later accretion of gas carries high angular momentum (e.g., Kimm et al. 2011; Stewart et al. 2011); therefore, it redefines the rotational axis of the system.

More explicitly, Figure 11 shows how the circularity parameters of the stars change over time as the galaxy develops a counter-rotating disk. The first column shows the distribution of the stars of the progenitors of the galaxy at $z=1.4,1.0$, and 0.7 in the plane of circularity versus distance. The color of each star represents its formation redshift. As visual guides, we also trace the evolution of 20 randomly selected stars formed at $z=1.4$ (yellow-green circles) and at $z=1.0$ (light blue stars) on the phase-space diagrams. The PDFs of the circularity parameters of stars in the progenitors, shown in the second column, are colored according to their average formation redshift. The third and fourth columns show edge-on $r$-band images of the progenitors and the $r$-band flux-weighted line-ofsight velocity map. The yellow-green and light blue arrows show the scaled projected angular momentum of the 20 selected stars formed at $z=1.4$ and $z=1.0$, respectively.

The stars formed at $z=1.4$ (the yellow-green circles in the top panel) are primarily disk stars at birth (most of them have circularity parameters higher than 0.5). Therefore, the circularity parameters display a gradual age dependence, with younger stars having higher circularity (see the color variation in the right PDF). Their angular momentum direction is mostly aligned with the galactic rotational axis. Also, the morphology at $z=1.4$ is fairly disky with $D / T \sim 0.4$, as is also seen in the velocity map.

At $z=1.0$ (middle panels), after the merger, most of the stars formed at $z=1.4$ (yellow-green) migrate to the spheroidal component (with lower $\epsilon$ ). Meanwhile, the galaxy produces counter-rotating stars with $\epsilon \sim-1$ (light blue stars), as the star-forming gas rotates around an axis opposite to the galactic spin axis ${ }^{15}(\cos \alpha \sim-1$ in Figure $10(d))$. These young stars start to build a small peak on the negative side of the circularity PDF (second column). Also, they have much higher angular momentum with direction opposite to that of the galactic rotational axis (see the light blue arrows at $z=1.0$ ), compared to the old stars formed at $z=1.4$ (the yellow-green arrows). Thus, the $r$-band-weighted velocity map shows a counter-rotation in the outer parts due to the young luminous stars. Conversely, the central parts, where pre-existing stars are dominant, still exhibit a weak corotation.

As the galaxy continues to produce these counter-rotating stars, the galactic rotational axis is reversed and aligned with the rotational axis of the gas. At $z=0.7$ (bottom panels), the counter-rotating stars formed at $z=1.0$ (light blue stars) have positive circularity, mostly higher than 0.7 , with a significant peak at $\epsilon \sim 1$ with young stars (blue) in the PDF. The angular momentum direction of these stars has finally aligned with the galactic rotational axis, and they show clear (co)rotation in the velocity map. Interestingly, stars located away from the disk plane, which are most likely to be old stars formed before $z=1.0$, show a mild counter-rotation. Even though the young stars contribute significantly to determining the axis of galactic rotation with higher angular momentum, $D / T$ of the galaxy measured by mass is not sufficiently high for it to be classified as disk-dominated, because these young stars do not outnumber the pre-existing stars, most of which are spheroid stars.

\section{Discussion}

\subsection{Evolution of Kinematic Morphology}

In this section, we explore the evolution of galaxies with different kinematic morphology (final disk-to-total ratio). We selected disk-dominated, intermediate, and spheroid-dominated galaxies at $z=0.7$ and tracked their kinematic morphological evolution back to $z=4$, which is shown in the left panels of Figure 12. In addition to the average growth of $D / T$ in the three groups, we also added the evolution of $D / T$ of individual galaxies as specimens of massive and low-mass diskdominated ("A," "B"), intermediate ("C," "D"), and spheroiddominated galaxies ("E," "F"). The right panels of Figure 12 show the $r$-band edge-on images of those specimens at $z=3.0$, 2.0, 1.0, and 0.7 .

At $z \sim 3$, the disk component is still negligible in all of our galaxies, resulting in $D / T$ between 0.2 and 0.3 . Diskdominated galaxies (selected at $z=0.7$ ), however, keep growing their disks until $z=0.7$. The increase in the disk component is more pronounced in massive galaxies than in low-mass ones. As illustrated in Figure 12, the massive galaxy ("A") has a well-developed disk from $z \sim 2$, while the lowmass galaxy ("B") has one from approximately $z \sim 1$.

Low-mass spheroid-dominated galaxies ("F"), conversely, have not been able to predominantly form disk stars, and

\footnotetext{
15 Keep in mind that the galactic rotational axis is determined by all the stars within $R_{90}$, the spherically averaged radius containing $90 \%$ of the stars in a galaxy.
} 

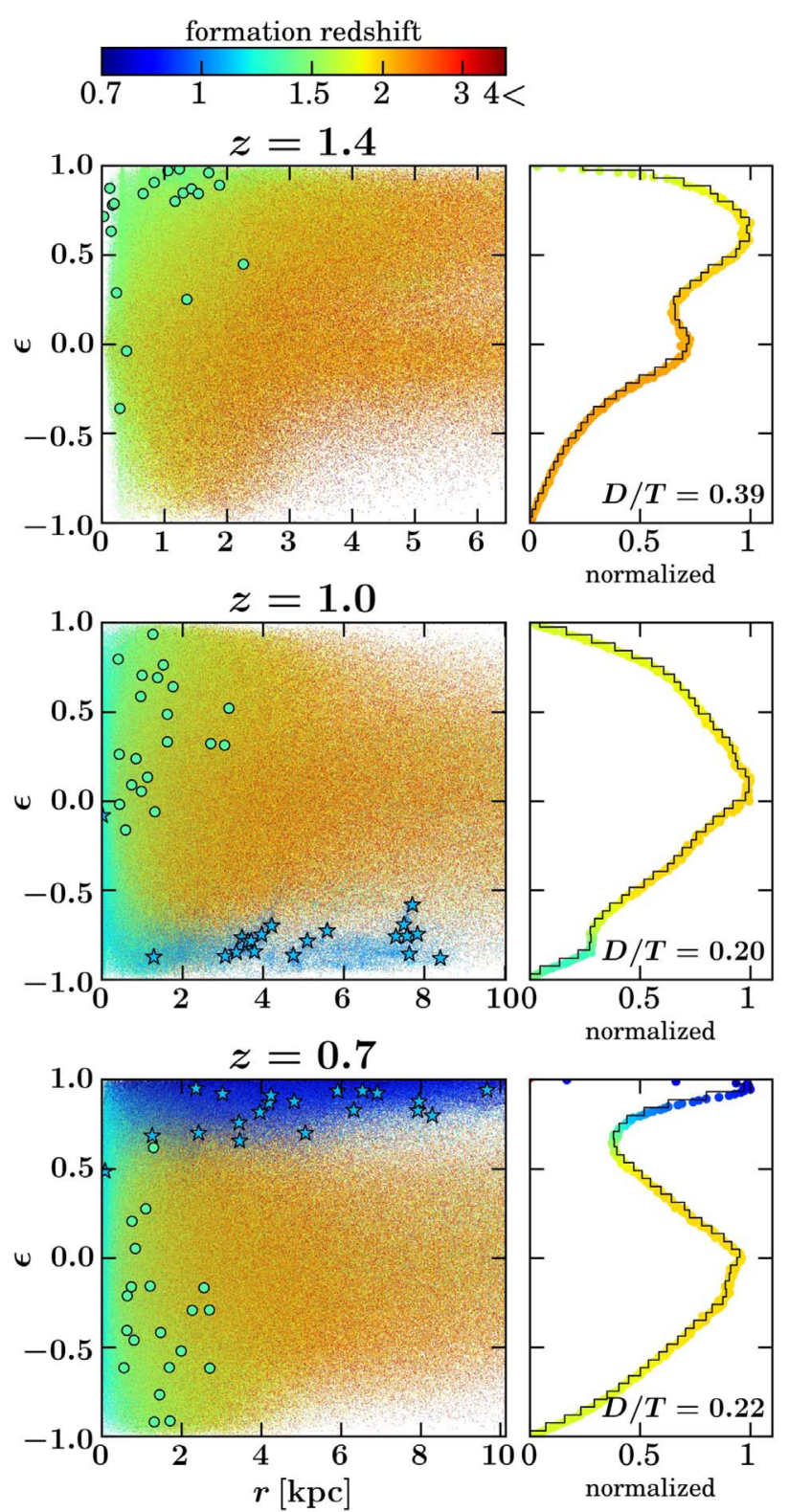
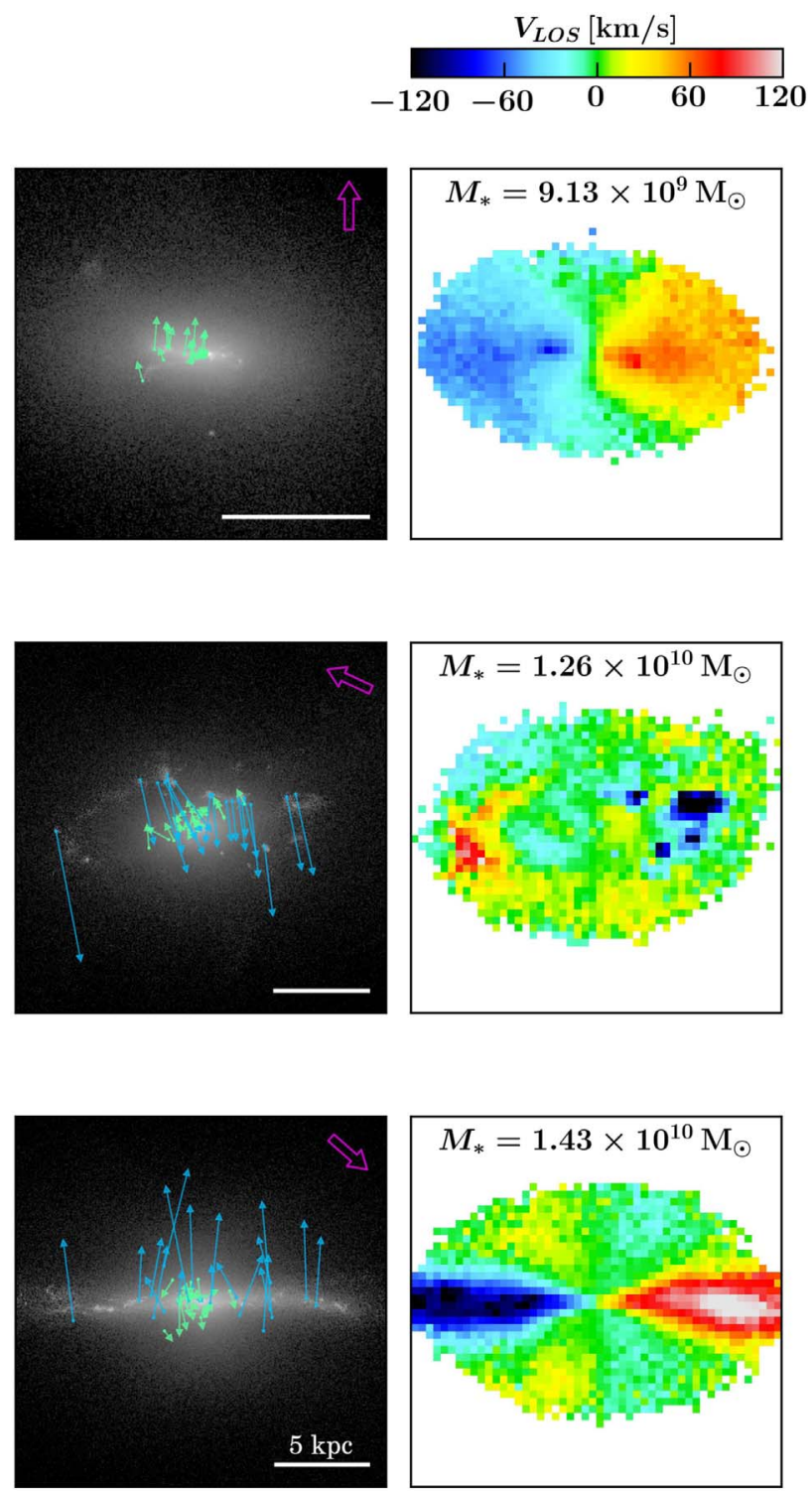

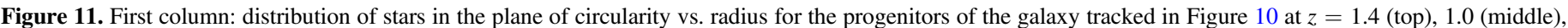

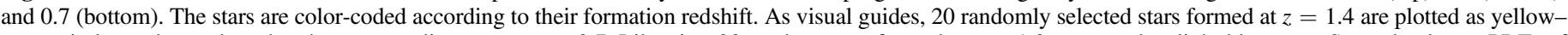

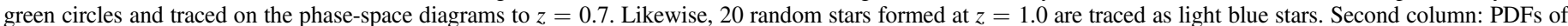

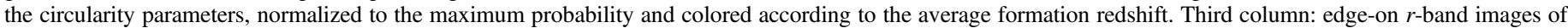

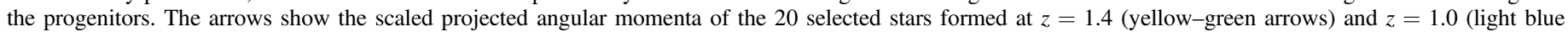

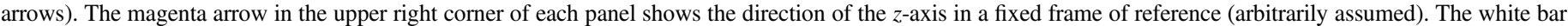
represents $5 \mathrm{kpc}$ (physical scale). Fourth column: the $r$-band flux-weighted line-of-sight velocity map.

therefore have continually had low $D / T$. In our sample, the massive spheroid-dominated galaxies ("E" as an example) seem to be formed via severe disk disruption (Figure 9(c)) and high accretion (Figure 8(a)); therefore, some of them once had well-developed disks with peaks in the $D / T$ evolution. Indeed, as a visual inspection of the right panel shows, the sample galaxy "E" developed a disk at $z \sim 2$, only to be disrupted and become a spheroid-dominated galaxy at $z=0.7$. Some galaxies develop counter-rotating disks, as mentioned in Section 4.4. Unfortunately, we lack massive spheroids in the sample because our galaxies are confined to a field environment where dramatic merger-driven morphological transformations are rare.

\subsection{Contribution from Different Channels to Disk and Spheroidal Components}

The top panel of Figure 13 presents a general picture of the evolution of galaxies from an extrapolation based on Section 4; galaxies grow via in situ star formation and accretion (of stars that are formed ex situ). In the early stage of galaxy formation, stars mostly form with misaligned orbits (orange) through numerous mergers between protogalaxies. As redshift decreases, most stars are formed with aligned orbits (blue), though the star formation rate is decreasing. As introduced in Figure 1, we divide the possible channels that contribute to the disk and spheroidal components of a galaxy. Let us quantify 

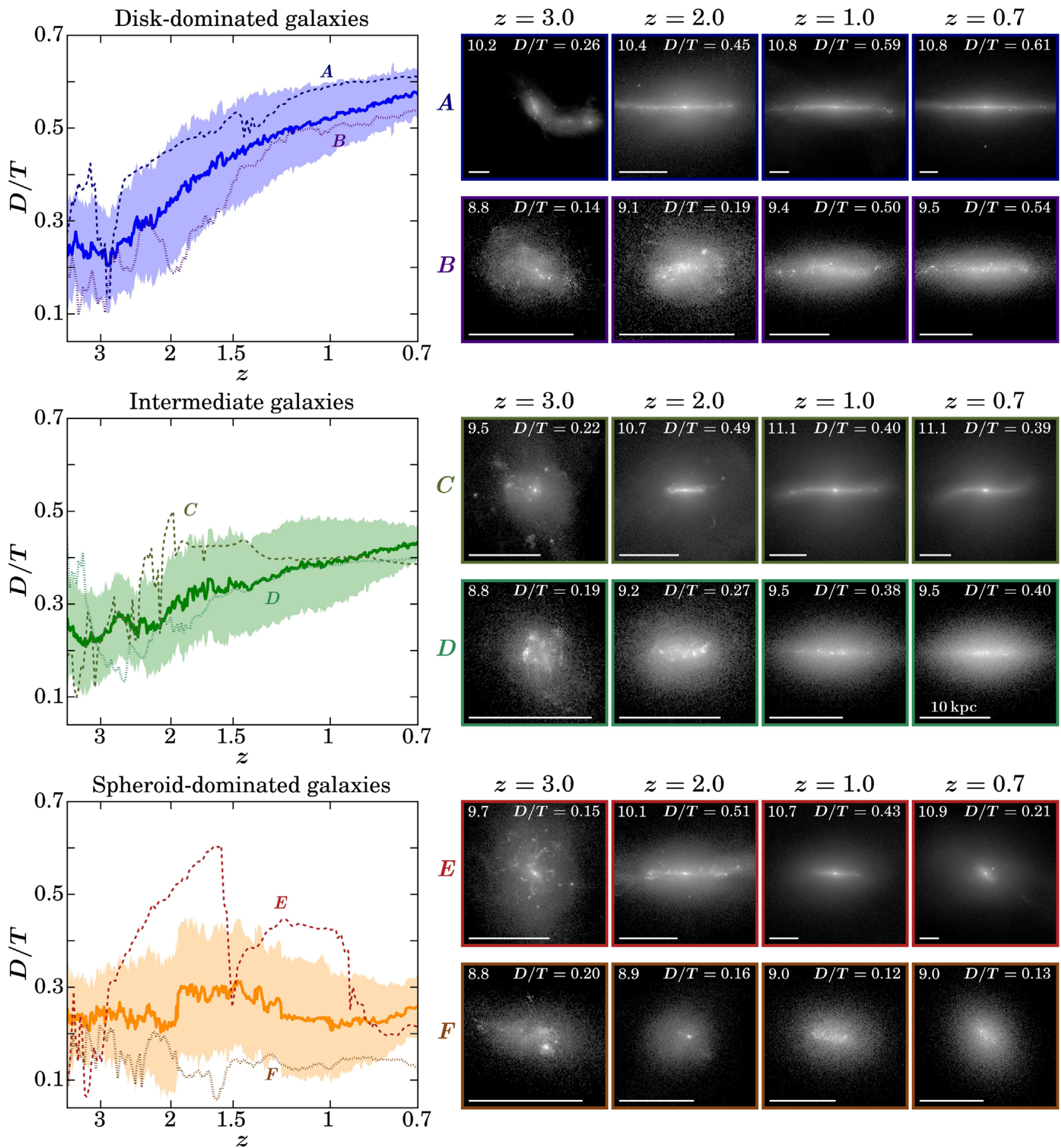

Figure 12. Left: $D / T$ evolution of galaxies with different morphologies. The solid lines indicate the average $D / T$ of the disk-dominated (blue line), intermediate (green line), and spheroid-dominated (orange line) galaxies, and the shading represents $1 \sigma$. We also plot the $D / T$ evolution of individual galaxies as specimens of different galaxy types: massive disk-dominated ("A," dashed dark blue line), low-mass disk-dominated ("B," dotted purple line), massive intermediate ("C," dashed dark green line), low-mass intermediate ("D," dotted green line), massive spheroid-dominated ("E," dashed dark red line), and low-mass spheroid-dominated ("F," dotted brown line). Right: $r$-band (in rest frame) edge-on images of those specimens from $z=3.0$ to $z=0.7$.

the importance of these channels and revisit the different kinematic morphology of galaxies (probed by disk-to-total ratio). The bottom of Figure 13 provides the estimates (as percentages) of stars with different origins in the galaxies. The numbers shown in blue and orange colors are the average estimates for the 53 disk-dominated galaxies $(D / T>0.5)$ and the 43 spheroid-dominated galaxies $(D / T<0.35)$ selected at $z=0.7$. Table 1 also presents the estimates averaged over the massive $\left(>10^{10} M_{\odot}\right)$ and the low-mass $\left(10^{9}-10^{10} M_{\odot}\right)$ galaxies and the associated standard deviation. Based on the estimates, we summarize the formation of galaxies with different kinematic morphology (disk-to-total ratio) as follows.

Disk-dominated galaxies: approximately $90 \%$ of the stars in the disk-dominated galaxies are formed in situ, and many of them $\left(64.8 \%\right.$ in total, $\left.f_{\text {disk }}^{\text {at birth }} \sim 0.74\right)$ are born with aligned orbits with respect to the rotating plane. We also found that there is a mass dependence for galaxies to start disk-mode star formation (Sections 4.2). Therefore, higher fractions of stars are 
high z

low z

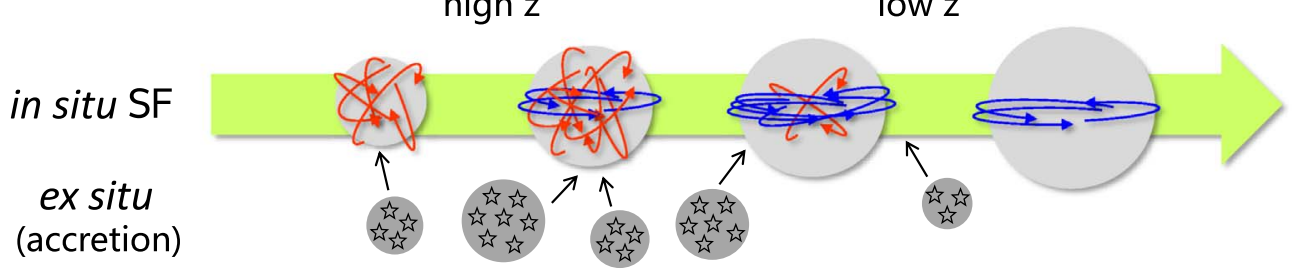

Origins of stellar particles

Morphological components

at $\mathrm{z}=0.7$

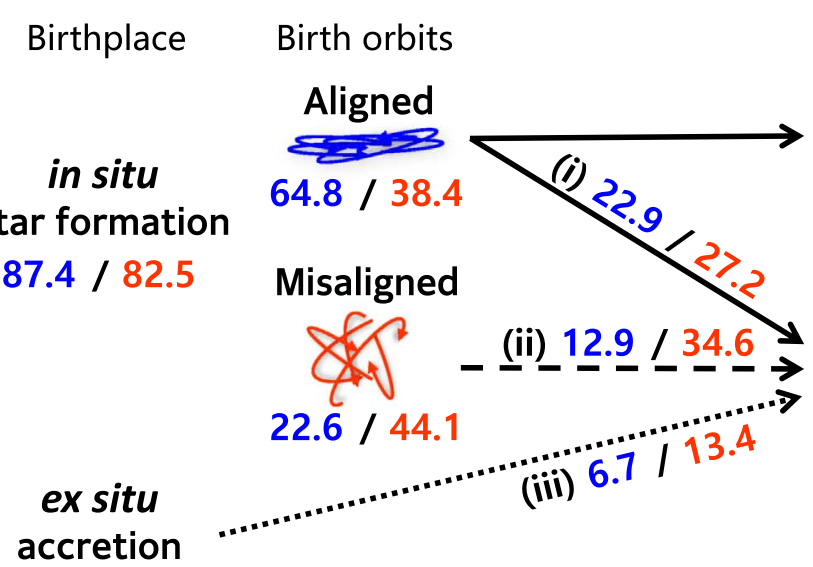

\section{Disk}

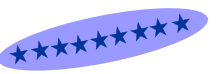

$57.5 / 24.8$

Spheroid

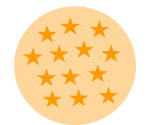

$42.5 / 75.2$
Kinematic morphology

$(\mathrm{D} / \mathrm{T})$

\section{$12.6 / 17.5$}

Figure 13. Top: a general picture of the evolution of a galaxy based on the discussion of Section 4.2. Galaxies grow from in situ star formation and accretion of stars that are formed ex situ. As redshift decreases, stars are more likely to form with aligned orbits (blue orbits). Bottom: a schematic diagram showing the estimates (as percentages) of stellar particles with different origins in the disk and spheroidal components (same as Figure 1). Disk and spheroidal components of galaxies are kinematically decomposed at $z=0.7$, and the arrows indicate the different channels to the spheroids: (i) stars initially formed in the disk ("aligned" initial orbits) yet migrated to the spheroid, (ii) stars born with "misaligned" initial orbits, and (iii) accreted stars. The numbers shown in blue and orange colors are the average estimates for the 53 disk-dominated galaxies $(D / T>0.5)$ and the 43 spheroid-dominated galaxies $(D / T<0.35)$, respectively.

Table 1

Stellar Origins of the Galaxies with Different Mass and Morphology

\begin{tabular}{|c|c|c|c|c|c|c|c|}
\hline & \multicolumn{3}{|c|}{ Origins of stellar particles } & \multicolumn{4}{|c|}{ Morphological components } \\
\hline & \multicolumn{2}{|c|}{ in situ } & \multirow{2}{*}{ ex situ } & \multirow{2}{*}{ Disk } & \multicolumn{3}{|c|}{ Spheroid ((i) + (ii) + (iii)) } \\
\hline & Aligned $\left(f_{\text {disk }}^{\text {at birth }}\right)$ & Misaligned & & & (i) & (ii) & (iii) \\
\hline Disk-dominated (53) & $64.8 \pm 9.1(0.74)$ & $22.6 \pm 7.1$ & $12.6 \pm 8.6$ & $57.5 \pm 5.2$ & $22.9 \pm 4.4$ & $12.9 \pm 4.2$ & $6.7 \pm 4.7$ \\
\hline Massive disk (15) & $66.5 \pm 7.4(0.82)$ & $14.8 \pm 4.3$ & $18.7 \pm 8.2$ & $59.3 \pm 5.2$ & $19.9 \pm 4.3$ & $9.4 \pm 2.7$ & $11.4 \pm 5.2$ \\
\hline Low-mass disk (38) & $64.1 \pm 9.6(0.71)$ & $25.7 \pm 5.4$ & $10.2 \pm 7.5$ & $56.8 \pm 5.0$ & $24.1 \pm 3.8$ & $14.3 \pm 3.9$ & $4.9 \pm 2.8$ \\
\hline Spheroid-dominated (43) & $38.4 \pm 10.8(0.47)$ & $44.1 \pm 13.4$ & $17.5 \pm 12.4$ & $24.8 \pm 6.6$ & $27.2 \pm 8.1$ & $34.6 \pm 11.4$ & $13.4 \pm 9.2$ \\
\hline Massive spheroid (5) & $41.1 \pm 8.6(0.61)$ & $26.5 \pm 8.0$ & $32.4 \pm 7.8$ & $27.8 \pm 5.5$ & $30.8 \pm 11.0$ & $19.8 \pm 4.8$ & $21.7 \pm 5.7$ \\
\hline Low-mass spheroid (38) & $38.0 \pm 11.0(0.45)$ & $46.4 \pm 12.1$ & $15.5 \pm 11.5$ & $24.4 \pm 6.6$ & $26.7 \pm 7.5$ & $36.6 \pm 10.5$ & $12.3 \pm 9.0$ \\
\hline All (144) & $54.2 \pm 14.4(0.62)$ & $32.4 \pm 13.0$ & $13.3 \pm 10.4$ & $42.8 \pm 14.3$ & $26.0 \pm 6.6$ & $22.2 \pm 11.5$ & $9.0 \pm 7.6$ \\
\hline
\end{tabular}

Note. The estimates (as percentages) of the origins of stellar particles (in situ and ex situ) in the galaxies and in the morphological components, disk and spheroid (see Figure 13). Each row presents the estimates averaged for disk-/spheroid-dominated galaxies (53 and 43 galaxies, respectively) and for all the galaxies with masses greater than $10^{9} M_{\odot}(144$ galaxies, selected at $z=0.7)$ and the associated standard deviation. Note that all these quantitative estimates are highly sensitive to the definition of disk and spheroidal components (and also to the definition of aligned and misaligned initial orbits).

born as disk stars in heavier galaxies $\left(f_{\text {disk }}^{\text {at birth }} \sim 0.82\right)$ than in lower-mass galaxies $\left(f_{\text {disk }}^{\text {at birth }} \sim 0.71\right)$. Some of the disk-born stars (22.9\% in total, solid arrow) become part of the spheroids as their orbits are perturbed; therefore, approximately half of the spheroidal components originate from the disks (Section 4.3). Finally, $12.6 \%$ of the stars in disk-dominated galaxies are formed ex situ and around a half of those (or $6.7 \%$ in total, dotted arrow) contribute to the growth of the spheroids.
Spheroid-dominated galaxies: unlike disk-dominated galaxies, many of the stars in spheroid-dominated galaxies are born with misaligned orbits ( $44.1 \%$ in total, $f_{\text {disk }}^{\text {at birt }} \sim 0.47$ ) and contribute to the spheroidal components (dashed arrow). Massive galaxies have a higher fraction of disk stars at birth $\left(f_{\text {disk }}^{\text {at birth }} \sim 0.61\right)$ than low-mass galaxies $\left(f_{\text {disk }}^{\text {at birth }} \sim 0.45\right.$ ); however, most of the disk stars eventually migrate to the spheroidal components (see Figure 9(c)). Indeed, the transformation from disks to spheroids 
((i), solid arrow) is the most important channel (30.8\% in total) for growing the spheroidal components in massive galaxies. On average, spheroid-dominated galaxies have higher accretion levels (17.5\% in total) than disk-dominated galaxies, a trend that is more pronounced for massive galaxies (see also Figure 8(a)). In addition, a higher fraction of ex situ stars contribute to the spheroidal components.

\subsection{Caveats}

Throughout this study, we used a simple circularity cutoff of $\epsilon=0.5$ (or $\epsilon_{\text {birth }}=0.5$ ) for identifying disk (or disk-born) stars. In addition, our study suffers from the fact that the New Horizon simulation has ended at $z=0.7$, covering only half of cosmic history. While there is no absolute criterion to decompose a galaxy into disk and spheroid, we discuss some limitations that may arise from applying this simple cutoff and exploring only field galaxies down to $z=0.7$.

In New Horizon at $z=0.7$, even the galaxy with the highest value of $D / T$ (which is $\sim 0.7$, see Figure 4 ) has a considerable spheroidal component $(\sim 0.3)$. The absence of pure kinematic disk galaxies in our sample partially arises from the shortcoming of our kinematic decomposition method; we consider all the stars with $\epsilon<0.5$ to be part of the spheroid. This means that even if all the stars of a galaxy followed a single distribution function, strongly peaked at $\epsilon=1.0$, its $\epsilon<0.5$ tail would be counted as part of a spheroidal component. Similarly, disk components also have contamination from spheroids, especially in spheroid-dominated galaxies. If we assume a Gaussian distribution of the circularity parameter for the bulk of randomly orbiting stars, the stars in the right tail above $\epsilon>0.5$ are classified as disk stars.

The fact that our sample is biased to the field environment and explored only the first half of cosmic history is another issue we should address. We have discussed in Section 3.2 that this might lead to the discrepancy in the morphological mix between the New Horizon and the local galaxies: the New Horizon lacks massive elliptical galaxies. In a denser environment such as clusters, the majority of massive galaxies are early-type even at $z \sim 0.7$ (e.g., Smith et al. 2005; van der Wel et al. 2007). From a quantitative point of view, the importance of ex situ (accreted) stars (described by the dotted arrow (iii) in Figure 13) in cluster galaxies would be heightened compared to the New Horizon sample.

To sum up, the quantitative description of the channels discussed in Section 5.2 is therefore highly sensitive to the definition of the disk and spheroid. Nonetheless, we assumed that the consistent cutoff of $\epsilon=0.5$ is reasonable enough to disentangle the disk and spheroidal components of highredshift galaxies undergoing dramatic evolution. Given that the vast majority of galaxies in the local universe are located in the field, the conclusions drawn from our sample should still hold for the bulk of the galaxy population. While the estimates we provide may not be suitable for direct comparison with the local universe, we expect these to be the guiding values for those from future high-resolution large-volume simulations down to $z=0$ and observational studies.

\section{Conclusions}

Using the New Horizon simulation, we explored the origin of disk and spheroidal components of 144 field galaxies with masses greater than $10^{9} M_{\odot}$. We decomposed the simulated galaxies into disks and spheroids based on the orbital properties of their stellar particles. We traced the origins of stellar particles according to where they formed and the properties of their orbits at birth (see Section 4.1). Our main results can be summarized as follows.

1. Galaxies form disk stars in a mass-dependent way; massive galaxies $\left(M_{*, \mathrm{z}}=0.7>10^{10} M_{\odot}\right)$ start to develop their disks at $z \sim 1-2$, while low-mass galaxies $\left(10^{9} M_{\odot}\right.$ $<M_{*, \mathrm{z}=0.7}<10^{10} M_{\odot}$ ) do so at $z<1$ (Section 4.2 and see also Y. Dubois et al. 2019, in preparation). Indeed, the fraction of disk stars at birth ( $\left.f_{\text {disk }}^{\text {at birth }}\right)$ increases with stellar mass; most of the stars in massive galaxies formed in the disks $\left(f_{\text {disk }}^{\text {at birth }} \sim 0.76\right.$ ), whereas a half of the low-mass galaxies in our sample have not yet developed their disks by $z \sim 0.7$. The formation of disks is affected by accretion as well; galaxies with higher accretion have lower $f_{\text {disk }}^{\text {at birth }}$, because mergers or fly-bys can boost gas turbulence in galaxies, making galaxies less likely to form corotating disk stars. Therefore, the fraction of disk stars at birth depends on both mass and accretion history (Figure 8).

2. Stars with disk-origin contribute significantly to the spheroidal components except for the low-mass spheroiddominated population, because they have not formed many disk stars by $z \sim 0.7$ (Figure 9). In the disk-dominated galaxies (both massive and low-mass), these stars with disk-origin make up around half of their spheroidal component. Accretion plays an important role in the spheroidal components in massive galaxies, especially massive spheroid-dominated galaxies (accounting for $\sim 30 \%$ of the spheroid stars).

3. The development of a counter-rotating disk component is not very rare in the evolution of massive galaxies; three of 24 massive galaxies $(12.5 \%)$ in our sample have had such structures. These counter-rotating structures can last for more than a gigayear, until they become the dominant component and flip the angular momentum of the galaxy in the opposite direction. As a result, kinematically decoupled features (with old stars counter-rotating in the inner regions while young stars formed recently corotate) appear on (edge-on) velocity maps (Figure 11).

In conclusion, disk and spheroidal components of galaxies are formed via several processes, and the kinematic morphology of galaxies (i.e., disk-to-total ratio) is determined by the significance of each process. Because the importance of the processes (e.g., disk formation, migration of disk stars to spheroidal components, and accretion of disrupted satellite stars) depends on both stellar mass and accretion history, the morphology of galaxies also needs to be understood in relation to them.

Identifying the origin and kinematics of individual stars in a galaxy is extremely challenging in observations. Only in the Milky Way has it been possible to obtain kinematic information on individual stars, e.g., via RAVE (Steinmetz et al. 2006), Gaia (Gaia Collaboration 2016), and APOGEE (Majewski et al. 2017). The kinematic properties of external galaxies are primarily studied with integral field unit surveys (Cappellari et al. 2007; Emsellem et al. 2007; Croom et al. 2012; Sánchez et al. 2012; Bundy et al. 2015), more actively at lower redshifts. We expect that future observations will provide more detailed information on the origins of morphological components in galaxies at high redshifts, too. 
We thank the referee for constructive comments. S.K.Y., the corresponding author, acknowledges support from the Korean National Research Foundation (NRF-2017R1A2A05001116). This research was supported in part by the National Science Foundation under grant No. NSF PHY-1748958. This work was granted access to the high-performance computing resources of CINES under the allocations c2016047637 and A0020407637 from GENCI, and KISTI (KSC-2017-G2-0003). Large data transfer was supported by KREONET, which is managed and operated by KISTI. This work relied on the HPC resources of the Horizon Cluster hosted by Institut d'Astrophysique de Paris. We warmly thank S. Rouberol for running the cluster on which the simulation was post-processed. T.K. was supported by the Yonsei University Future-leading Research Initiative (RMS2-2018-220183). H.C. acknowledges the support by Norwegian Research Council Young Research Talents Grant 276043 Simulating the Circumgalactic Medium and the Cycle of Baryons in and out of Galaxies throughout Cosmic History The research of J.D. is supported by Adrian Beecroft and STFC. M.V. acknowledges funding from the European Research Council under the European Community's Seventh Framework Programme (FP7/2007-2013 grant Agreement no. 614199, project "BLACK"). S.K. acknowledges a Senior Research Fellowship from Worcester College Oxford. This work is partially supported by the Spin(e) grant ANR-13- BS05-0005 of the French Agence Nationale de la Recherche, and ERC grant 670193.

\section{ORCID iDs}

Min-Jung Park 누 https://orcid.org/0000-0002-8435-9402 Sukyoung K. Yi (i) https://orcid.org/0000-0002-4556-2619 Taysun Kimm (1) https://orcid.org/0000-0002-3950-3997 Hoseung Choi (i) https://orcid.org/0000-0001-7229-0033 Marta Volonteri iㅏ https://orcid.org/0000-0002-3216-1322

\section{References}

Abadi, M. G., Navarro, J. F., Steinmetz, M., \& Eke, V. R. 2003, ApJ, 597, 21 Athanassoula, E. 2005, MNRAS, 358, 1477

Aubert, D., Pichon, C., \& Colombi, S. 2004, MNRAS, 352, 376

Aumer, M., \& White, S. D. 2013, MNRAS, 428, 1055

Aumer, M., White, S. D., \& Naab, T. 2014, MNRAS, 441, 3679

Barnes, J. E. 2002, MNRAS, 333, 481

Benson, A. J., Džanović, D., Frenk, C. S., \& Sharples, R. 2007, MNRAS, 379, 841 Bondi, H., \& Hoyle, F. 1944, MNRAS, 104, 273

Bruzual, G., \& Charlot, S. 2003, MNRAS, 344, 1000

Bundy, K., Bershady, M. A., Law, D. R., et al. 2015, ApJ, 798, 7

Cappellari, M., Emsellem, E., Bacon, R., et al. 2007, MNRAS, 379, 418

Chabrier, G. 2005, Astrophysics and Space Science Library, 327, 41

Clauwens, B., Schaye, J., Franx, M., \& Bower, R. G. 2018, MNRAS, 478, 3994

Cole, S., Lacey, C., Baugh, C., \& Frenk, C. 2000, MNRAS, 319, 168

Conselice, C. J. 2006, MNRAS, 373, 1389

Cooper, A. P., Parry, O. H., Lowing, B., Cole, S., \& Frenk, C. 2015, MNRAS, 454, 3185

Croom, S. M., Lawrence, J. S., Bland-Hawthorn, J., et al. 2012, MNRAS, 421, 872

Dekel, A., Sari, R., \& Ceverino, D. 2009, ApJ, 703, 785

Driver, S. P., Allen, P. D., Graham, A. W., et al. 2006, MNRAS, 368, 414

Dubois, Y., Devriendt, J., Slyz, A., \& Teyssier, R. 2012, MNRAS, 420, 2662

Dubois, Y., Peirani, S., Pichon, C., et al. 2016, MNRAS, 463, 3948

Dubois, Y., Pichon, C., Welker, C., et al. 2014a, MNRAS, 444, 1453

Dubois, Y., Volonteri, M., \& Silk, J. 2014b, MNRAS, 440, 1590

El-Badry, K., Quataert, E., Wetzel, A., et al. 2018, MNRAS, 473, 1930

Elmegreen, B. G., Bournaud, F., \& Elmegreen, D. M. 2008, ApJ, 688, 67

Emsellem, E., Cappellari, M., Krajnović, D., et al. 2007, MNRAS, 379, 401

Fall, S. M. E. G. 1980, MNRAS, 52, 189

Font, A. S., Mccarthy, I. G., Crain, R. A., et al. 2011, MNRAS, 416, 2802
Gaia Collaboration 2016, A\&A, 595, A2

Garrison-Kimmel, S., Hopkins, P. F., Wetzel, A., et al. 2018, MNRAS, 481,4133

Genel, S., Dekel, A., \& Cacciato, M. 2012, MNRAS, 425, 788

Governato, F., Brook, C. B., Brooks, A. M., et al. 2009, MNRAS, 398, 312 Haardt, F., \& Madau, P. 1996, ApJ, 461, 20

Hernquist, L. 1989, Natur, 340, 687

Hopkins, A. M., \& Beacom, J. F. 2006, ApJ, 651, 142

Hoyle, F., \& Lyttleton, R. A. 1939, MPCPS, 35, 405

Hubble, E. 1926, A\&A, 64, 321

Hung, C. L., Hayward, C. C., Yuan, T., et al. 2019, MNRAS, 482, 5125

Ilbert, O., Salvato, M., Le Floc'H, E., et al. 2010, ApJ, 709, 644

Johnson, H. L., Harrison, C. M., Swinbank, A. M., et al. 2018, MNRAS, 474, 5076

Johnston, E. J., Merrifield, M. R., Araǵon-Salamanca, A., \& Cappellari, M. 2013, MNRAS, 428, 1296

Kassin, S. A., Weiner, B. J., Faber, S. M., et al. 2012, ApJ, 758, 106

Kereš, D., Katz, N., Weinberg, D. H., \& Davé, R. 2005, MNRAS, 363, 2

Kimm, T., \& Cen, R. 2014, ApJ, 788, 121

Kimm, T., Devriendt, J., Slyz, A., et al. 2011, arXiv:1106.0538

Kimm, T., Katz, H., Haehnelt, M., et al. 2017, MNRAS, 466, 4826

Komatsu, E., Smith, K. M., Dunkley, J., et al. 2011, ApJS, 192, 18

Lee, J., \& Yi, S. K. 2017, ApJ, 836, 161

Majewski, S. R., Schiavon, R. P., Frinchaboy, P. M., \& Prieto, C. A. 2017, AJ, 154, 94

Marinacci, F., Pakmor, R., \& Springel, V. 2014, MNRAS, 437, 1750

Martig, M., Bournaud, F., Croton, D. J., Dekel, A., \& Teyssier, R. 2012, ApJ, 756,26

Martin, G., Kaviraj, S., Devriendt, J. E. G., Dubois, Y., \& Pichon, C. 2018, MNRAS, 480, 2266

Mckinney, J. C., Tchekhovskoy, A., \& Blandford, R. D. 2012, MNRAS, 423, 3083

Mo, H. J., Mao, S., \& White, S. D. 1998, MNRAS, 295, 319

Negroponte, J., \& White, S. D. M. 1983, MNRAS, 205, 1009

Noguchi, M. 1999, ApJ, 514, 77

Obreja, A., Dutton, A. A., Macciò, A. V., et al. 2018, MNRAS, 477, 4915

Obreja, A., Macciò, A. V., Moster, B., et al. 2019, MNRAS, 487, 4424

Ocvirk, P., Pichon, C., \& Teyssier, R. 2008, MNRAS, 390, 1326

Oser, L., Ostriker, J. P., Naab, T., Johansson, P. H., \& Burkert, A. 2010, ApJ, 725, 2312

Pichon, C., Pogosyan, D., Kimm, T., et al. 2011, MNRAS, 418, 2493

Pillepich, A., Nelson, D., Springel, V., et al. 2019, arXiv:1902.05553

Rodriguez-Gomez, V., Genel, S., Vogelsberger, M., et al. 2015, MNRAS, 449,49

Rodriguez-Gomez, V., Pillepich, A., Sales, L. V., et al. 2016, MNRAS, 458, 2371

Rosen, A., \& Bregman, J. N. 1995, ApJ, 440, 634

Sales, L. V., Navarro, J. F., Theuns, T., et al. 2012, MNRAS, 423, 1544

Sánchez, S. F. F., Kennicutt, R. C. C., Gil de Paz, A., et al. 2012, A\&A, 538, A8

Scannapieco, C., White, S. D. M., Springel, V., \& Tissera, P. B. 2009, MNRAS, 396, 696

Schmidt, M. 1959, ApJ, 129, 243

Searle, L., \& Zinn, R. 1978, A\&A, 225, 357

Simons, R. C., Kassin, S. A., Trump, J. R., et al. 2016, ApJ, 830, 14

Simons, R. C., Kassin, S. A., Weiner, B. J., et al. 2017, ApJ, 843, 46

Smith, G. P., Treu, T., Ellis, R. S., Moran, S. M., \& Dressler, A. 2005, ApJ, 620,78

Steinmetz, M., Zwitter, T., Siebert, A., et al. 2006, AJ, 132, 1645

Stewart, K. R., Kaufmann, T., Bullock, J. S., et al. 2011, ApJ, 738, 39

Sutherland, R. S., \& Dopita, M. A. 1993, ApJS, 88, 253

Teyssier, R. 2002, A\&A, 385, 337

Tissera, P. B., Scannapieco, C., Beers, T. C., \& Carollo, D. 2013, MNRAS, 432, 3391

Tissera, P. B., White, S. D., \& Scannapieco, C. 2012, MNRAS, 420, 255

Toomre, A., \& Toomre, J. 1972, ApJ, 178, 623

Trayford, J. W., Frenk, C. S., Theuns, T., Schaye, J., \& Correa, C. 2019, MNRAS, 483, 744

Turner, O. J., Cirasuolo, M., Harrison, C. M., et al. 2017, MNRAS, 471, 1280

Tweed, D., Devriendt, J., Blaizot, J., Colombi, S., \& Slyz, A. 2009, A\&A, 506, 647

van der Wel, A., Holden, B. P., Franx, M., et al. 2007, ApJ, 670, 206

White, S. D. M. 1984, ApJ, 286, 38

Zolotov, A., Dekel, A., Mandelker, N., et al. 2015, MNRAS, 450, 2327

Zolotov, A., Willman, B., Brooks, A. M., et al. 2009, ApJ, 702, 1058 